\title{
On the Autonomous Nemytskij Operator in Hölder Spaces
}

\author{
M. Goebel and F. Sachweh \\ Dedicated in gratitude to Lothar von Wolfersdorf on his 65 th birthday
}

\begin{abstract}
The paper is devoted to the autonomous Nemytskij operator (superposition operator) in Hölder spaces $H^{k+o}[a, b],(k, \alpha) \in \mathbb{Z}_{+} \times(0,1]$. We study acting, continuity, Lipschitz continuity, and Fréchet differentiability conditions. For $k=0, \alpha \in(0,1]$ and $k \in \mathbb{N}, \alpha=1$ the respective conditions are both necessary and sufficient. For $k \in \mathbb{N}, \alpha \in(0,1)$ only the acting condition is both necessary and sufficient; the other investigated properties are characterized by necessary and sufficient conditions different from each other.
\end{abstract}

Keywords: Hölder spaces, Lipschitz spaces, Nemytskij operator, superposition operator, acting conditions, boundedness, continuity and Lipschitz continuity, Fréchet differentiability

AMS subject classification: 47 H 30

\section{Introduction}

To describe the contents of this paper properly we start with introducing the function spaces needed in the following. by

Let $[a, b] \subset \mathbb{R}$ be a given bounded interval, $\alpha \in(0,1]$ and $h_{\alpha}: C[a, b] \rightarrow \overline{\mathbb{R}}$ defined

$$
h_{\alpha}(u)=\sup \left\{\frac{|u(t)-u(s)|}{|t-s|^{\alpha}} \mid t \neq s, t, s \in[a, b]\right\} .
$$

Then $u \in C[a, b]$ is said to be $\alpha$-Hölder continuous on $[a, b]$ if $h_{\alpha}(u)<\infty$. The set of all such functions is called (classical) Hölder space and denoted by $H^{\alpha}[a, b]$. Introducing the norm

$$
\|u\|_{H^{\circ}[a, b]}=\|u\|_{C[a, b]}+h_{\alpha}(u)
$$

the set $H^{\alpha}[a, b]$ becomes a Banach space. In the case of $\alpha=1$ we write Lip $[a, b]$ instead of $H^{1}[a, b]$; the elements of $L i p[a, b]$ are Lipschitz continuous functions on $[a, b]$. If further a natural number $k \in \mathbb{N}$ is given, then a function $u \in C^{k}[a, b]$ is said to be $(k, \alpha)$-Hölder continuous on $[a, b]$ if its $k$-th derivative $u^{(k)}$ is an $\alpha$-Hölder continuous

M. Goebel: Martin-Luther-Universität Halle-Wittenberg, FB Math. und Inform., D-06099 Halle (Saale); goebel@mathematik.uni-halle.de

F. Sachweh: Bispinckpl. 1-3, D-48683 Ahaus; f_sachweh@lauder.de 
function. The set of all such functions is denoted by $H^{k+\alpha}[a, b]$. When endowed with the norm

$$
\|u\|_{H^{k+\alpha}[a, b]}=\sum_{i=0}^{k}\left\|u^{(i)}\right\|_{C[a, b]}+h_{\alpha}\left(u^{(k)}\right)
$$

it is also a Banach space. Again, in the ase of $\alpha=1$ we use the notation $L_{i p} p^{k}[a, b]$ instead of $H^{k+1}[a, b]$. By convention, $H^{0+\alpha}[a, b]=H^{\alpha}[a, b]$ and $\operatorname{Lip}{ }^{0}[a, b]=\operatorname{Lip}[a, b]$ so that the space $H^{k+\alpha}\{a, b]$ is defined for any pair $(k, \alpha) \in \mathbb{Z}_{+} \times(0,1]$, where $\mathbb{Z}_{+}=\{0\} \cup \mathbb{N}$.

Now we turn to functions $f=f(x)$ acting in $\mathbb{R}$. For given $\alpha \in(0,1]$ a function $f \in C(\mathbb{R})$ is said to be locally $\alpha$-Hölder continuous on $\mathbb{R}$ if for each $x_{0} \in \mathbb{R}$ there is a positive number $\delta$ such that $f \in H^{\alpha}\left[x_{0}-\delta, x_{0}+\delta\right]$. This is equivalent to the condition $f \in H^{\alpha}[-r, r]$ for all positive numbers $r$. The symbol $H_{\text {loc }}^{\alpha}(\mathbb{R})$ denotes the set of all locally $\alpha$-Hölder continuous functions defined on $\mathbb{R}$. We write $f \in H_{\text {loc }}^{k+\alpha}(\mathbb{R})$, where again $k \geq 1$ is a natural number and $\alpha \in(0,1]$, provided $f \in C^{k}(\mathbb{R})$ and $f^{(k)} \in H_{\text {loc }}^{\alpha}(\mathbb{R})$. In the case of $\alpha=1$ we write $\operatorname{Lip}_{l o c}(\mathbb{R})$ and $L i p_{l o c}^{k}(\mathbb{R})$ instead of $H_{l o c}^{1}(\mathbb{R})$ and $H_{l o c}^{k+1}(\mathbb{R})$, respectively.

The subject of our paper is the autonomous Nemytskij operator $F u=f \circ u$, generated by a given function $f: \mathbb{R} \rightarrow \mathbb{R}$, as a map of the Hölder space $H^{k+\alpha}[a, b]$, $(k, \alpha) \in \mathbb{Z}_{+} \times(0,1]$, into itself. There are given conditions to $f$ ensuring $F$ acting in $H^{k+\alpha}\{a, b]$, boundedness of $F$, its continuity, local Lipschitz continuity and Fréchet differentiability, respectively. While for $H^{\alpha}[a, b], \alpha \in(0,1]$, and $\operatorname{Lip}^{k}[a, b]$, $k \in \mathbb{N}$, all respective conditions are both necessary and sufficient, for $H^{k+\alpha}[a, b]$ with $(k, \alpha) \in \mathbb{N} \times(0,1)$ this is only the case for the acting condition. Concerning the other properties for these parameters we have always separate necessary and sufficient conditions with a certain distinction between both.

The plan of the paper is as follows. In Section 2 we consider the case $k=0$, $\alpha \in(0,1]$, in Section 4 the case $k \in \mathbb{N}, \alpha=1$, and eventually in Section 5 the case $(\dot{k}, \alpha) \in \mathbb{N} \times(0,1)$. Section 3 is devoted to some preliminaries for the subsequent sections. Most of the results in Section 2 are known; the results in Sections 4 and 5 seem to be new. One of the purposes of this paper is to present a self-contained unified and not too sophisticated theory of the autonomous Nemytskij operator in $H^{k+\alpha}[a, b]$. Unfortunately, in Section 5 some questions remain open.

Dealing with nonlinear analysis means at first to study Nemytskij operators. We were led to the problems considered in this paper in studying an optimal control problem, in which the state equation is a nonlinear singular integral equation of Cauchy type. Concerning existence and uniqueness of solutions to the state equation we could refer to L. v. Wolfersdorf [26]. However, we needed also a simple and convenient condition ensuring e.g. differentiability of Nemytsjkij operators in Hölder spaces. First results suitable for our purposes can be found in M. Goebel [8]. An application of these results to the control problem just mentioned is given in $\mathrm{M}$. Goebel and D. Oestreich [10]. The material to be presented now is mainly based on F. Sachweh [21], which was written under the auspices of the other author. We have announced our results in $\mathrm{M}$. Goebel and F. Sachweh [11], where the reader can find a summary in form of a table (with an obvious misprint in the last row). 
From the papers dealing explicitly with autonomous Nemytskij operators in Hölder spaces we quote at the moment only M. Z. Berkolajko [4], P. Drábek [7] and M. Goebel [8]. In case of a non-autonomous Nemytskij operator the generating function $f$ depends on $t \in[a, b]$ and $x \in \mathbb{R}$. For such operators considered in a Hölder space $H^{\alpha}[a, b]$ the reader is referred to the papers $R$. Nugari $[19,20]$, R. Chiappinelli and R. Nugari [6], and M. Goebel [9], and in $H^{k+\alpha}[a, b]$ to T. Valent [24: Chapter II], E. Wegert [26: Section 1.3], and M. Lanza de Cristoforis [12]. Most of the quoted papers deal in spaces of functions depending on several variables. A profound account of all relevant literature for both autonomous and non-autonomous Nemytskij operators in different function spaces until about 1988 can be found in the monograph J. Appell and P. P. Zabrejko [2].

We finish this introductory section with some notations used throughout the whole paper. If $X$ is a Banach space, the set of all continuous operators from $X$ into itself is denoted by $C(X)$ and by $C^{1}(X)$ the subset of $C(X)$, whose elements are continuously Fréchet differentiable on $X$. Liploc $(X)$ denotes the set of all locally Lipschitz continuous operators acting in $X$ and $\mathcal{L}(X)$ the set of all linear bounded operators acting in $X$. The symbol $B\left(x_{0}, \delta\right) \subset X$ stands for an open ball in $X$ centered at $x_{0} \in X$ with radius $\delta>0$. For any function $f$ we denote its $i$-th derivative by $D^{i} f$ or $f^{(i)}$. Finally, $c$ denotes a generic constant.

\section{The Nemytskij operator in $H^{\alpha}[a, b]$}

Before starting to consider autonomous Nemytskij operators $F$ generated by 'f: $\mathbb{R} \rightarrow \mathbb{R}$ in the Hölder space $H^{\alpha}[a, b]$ with $\alpha \in(0,1]$, we give two well-known properties of this space in our first lemma.

Lemma 2.1.

(a) For any $u, v \in H^{\alpha}[a, b]$, $u v \in H^{\alpha}[a, b]$ and $\|u v\|_{H^{\alpha}[a, b]} \leq\|u\|_{H^{\alpha}[a, b]}\|v\|_{H^{a}[a, b]}$.

(b) For any $\beta \in(0, \alpha), H^{\alpha}[a, b] \subset H^{\beta}[a, b]$ with $h_{\beta}(u) \leq(b-a)^{\alpha-\beta} h_{\alpha}(u)$ for all $u \in H^{\alpha}[a, b]$ :

Our first theorem presents a necessary and sufficient acting condition proved by M. Z. Berkolajko [4] for generalized Hölder spaces. The proof of the necessity part given below is an simplified adaption of $P$. Drábek [7]. It is an easy task to derive a necessary and sufficient condition if acting of $F$ is combined with its boundedness as it was done e.g. by A. A. Babajev [3]. The subsequent corollary is an immediate consequence of Theorem 2.1 .

Theorem 2.1. The autonomous Nemytskij operator $F$ generated by $f$ maps $H^{\alpha}[a, b]$ into itself if and only if $f \in \operatorname{Lip}_{\text {loc }}(\mathbb{R})$.

Proof. It suffices to show the necessity of $f \in \operatorname{Lip}_{l o c}(\mathbb{R})$ provided $F$ is acting in $H^{\alpha}[a, b]$.

1. Let $x_{0} \in \mathbb{R}$ be arbitrarily fixed. The linear function $u_{0}$ defined by $u_{0}(t)=$ $t-a+x_{0}-\frac{b-a}{2}(t \in[a, b])$ belongs to $H^{\alpha}[a, b]$ and hence, by our assumption, there is a constant $L_{0}>0$ with

$$
\left|f\left(u_{0}(t)\right)-f\left(u_{0}(s)\right)\right| \leq L_{0}|t-s|^{\alpha} \quad(t, s \in[a, b])
$$


which implies

$$
|f(x)-f(y)| \leq L_{0}|x-y|^{\alpha} \quad\left(x, y \in B\left(x_{0}, \frac{b-a}{2}\right) \subset \mathbb{R}\right) .
$$

Consequently, $f \in H_{\text {loc }}^{\alpha}(\mathbb{R})$ and the statement is already proved for $\alpha=1$.

2. Let $\alpha \in(0,1)$. Assuming $f \notin L i p_{l o c}(\mathbb{R})$ we can find a number $r_{0}>0$ and two convergent sequences $\left(x_{n}\right),\left(y_{n}\right) \subset\left[-r_{0}, r_{0}\right], x_{n} \neq y_{n}$ for all $n \in \mathbb{N}$, such that

$$
\left|f\left(x_{n}\right)-f\left(y_{n}\right)\right|>n\left|x_{n}-y_{n}\right| \quad(n \in \mathbb{N}) \text {. }
$$

Because of the first point of the proof, the left-hand side of $(2.1)$ is bounded and so $\left(x_{n}\right)$ and $\left(y_{n}\right)$ have the same limit, say $x_{0}$. We put

$$
B_{n}=B\left(x_{0}, \gamma_{n+1}\right) \subset \mathbb{R}, \quad \text { where } \gamma_{n}=\frac{b-a}{2^{n_{0}+n+2}} \quad(n \in \mathbb{N}),
$$

with $n_{0} \in \mathbb{N}$ so large that $\gamma_{n}<1$ for all $n \in \mathbb{N}$. Then for every $n \in \mathbb{N}$ there is a $k_{n} \in \mathbb{N}$ with $x_{k_{n}}, y_{k_{n}} \in B_{n}$ and hence $\left|x_{k_{n}}-y_{k_{n}}\right|<2 \gamma_{n+1}=\gamma_{n}$ for all $n \in \mathbb{N}$. We may assume $k_{n}>k_{m}$ for $n>m$. By setting $\delta_{n}=\left|x_{k_{n}}-y_{k_{n}}\right|^{1 / \alpha} \quad(n \in \mathbb{N})$ we get another sequence $\delta_{n} \subset(0,1)$ with $\delta_{n}<\gamma_{n}$. Now we choose a number $t_{1} \in\left(a, a+\frac{b-a}{4}\right)$ and define

$$
t_{n}=t_{1}+\sum_{i=1}^{n-1}\left(\gamma_{i}+\delta_{i}\right) \quad \text { and } \quad s_{n}=t_{n}+\delta_{n} \quad(n \in \mathbb{N}) \text {. }
$$

Obviously, $\left(t_{n}\right),\left(s_{n}\right) \subset[a, b]$ and $\gamma_{m}<t_{n}-t_{m}$ for arbitrary $m, n \in \mathbb{N}$ with $n>m$. Since $0<t \leq 1$ implies $t \leq t^{\alpha}$ and $t>1$ implies $t^{\alpha}>1$, we find $\gamma_{m}<\left(t_{n}-t_{m}\right)^{\alpha}$ and in the same way also $\gamma_{m}<\left(t_{n}-s_{m}\right)^{\alpha}$ and $\gamma_{m}<\left(s_{n}-s_{m}\right)^{\alpha}$ for all $m, n \in \mathbb{N}$ with $n>m$. We introduce on $M=\left\{t_{n}\right\}_{n \in \mathbf{N}} \cup\left\{s_{n}\right\}_{n \in N}$ a function $u=u(t)$ by setting

and claim

$$
u(t)= \begin{cases}x_{k_{n}} & \text { for } t=t_{n} \\ y_{k_{n}} & \text { for } t=s_{n}\end{cases}
$$

$$
|u(t)-u(s)| \leq|t-s|^{\alpha} \quad(t, s \in M) .
$$

Indeed, let $m, n \in \mathbb{N}$ with $n>m$. Then we have

$$
\left|u\left(t_{n}\right)-u\left(t_{m}\right)\right|=\left|x_{k_{n}}-x_{k_{m}}\right| \leq \gamma_{n+1}+\gamma_{m+1} \leq \gamma_{m} \leq\left(t_{n}-t_{m}\right)^{\alpha}
$$

and, analogously,

$$
\left|u\left(t_{n}\right)-u\left(s_{m}\right)\right| \leq\left(t_{n}-s_{m}\right)^{\alpha} \quad \text { and } \quad\left|u\left(s_{n}\right)-u\left(s_{m}\right)\right| \leq\left(s_{n}-s_{m}\right)^{\alpha} .
$$

If $m=n$, then

$$
\left|u\left(t_{n}\right)-u\left(s_{n}\right)\right|=\left|x_{k_{n}}-y_{k_{n}}\right|=\delta_{n}^{\alpha}=\left(s_{n}-t_{n}\right)^{\alpha} .
$$

Thereby (2.2) is proved. Due to E. J. McShane [18] (compare also J.-E. Björk [5] and W. Walter [24: p. 65]) $u$ can be extented to $[a, b]$ preserving the Hölder condition (2.2). This extension is again denoted by $u$. Then because of our assumption there exists a positive constant $L_{u}$ such that

$$
|f(u(t))-f(u(s))| \leq L_{u}|t-s|^{\alpha} \quad(t, s \in[a, b]) .
$$

For $t=t_{n}$ and $s=s_{n}$ this results in

$$
\left|f\left(x_{k_{n}}\right)-f\left(y_{k_{n}}\right)\right| \leq L_{u}\left|t_{n}-s_{n}\right|^{\alpha}=L_{u} \delta_{n}^{\alpha}=L_{u}\left|x_{k_{n}}-y_{k_{n}}\right| \text {. }
$$

Taking advantage of (2.1) we come to $L_{u} \geq k_{n}$ for all $n \in \mathbb{N}$. Since this is impossible the assertion is proved 
Corollary 2.1. Each autonomous Nemytskij operator acting in $H^{\alpha}[a, b]$ is bounded.

Unfortunately, not each Nemytskij operator acting in $H^{\alpha}[a, b]$ is continuous. As a simple example one may take $F$ generated by the function $f$, which is defined by $f(x)=$ $|x|(x \in \mathbb{R})$. Obviously, $F$ maps $H^{\alpha}[0,1]$ into itself. But this map is not continuous. To see this, take $u_{n}$ defined by $u_{n}(t)=t^{\alpha}-\varepsilon_{n}(t \in[0,1])$, with $\left(\varepsilon_{n}\right) \subset(0,2)$ and $\varepsilon_{n} \rightarrow 0$. Then $u_{n} \in H^{\alpha}[0,1]$ and $u_{n} \rightarrow u_{0}$ in $H^{\alpha}[0,1]$ as $n \rightarrow \infty$, where $u_{0}(t)=t^{\alpha} \quad(t \in[0,1])$. However, $h_{\alpha}\left(f \circ u_{n}-f \circ u_{0}\right) \geq 2$ for all $n \in \mathbb{N}$, such that $F u_{n} \rightarrow F u_{0}$ in $H^{\alpha}[0,1]$.

In the literature we can find also $f$ defined by $f(x)=\min \{x, 1\} \quad(x \in \mathbb{R})$ as another example for a function generating a non-continuous Nemytskij operator in $H^{\alpha}[0,1]$. But the given arguments are correct only for $\alpha=1$, i.e. in the space $\operatorname{Lip}[0,1]$, and false for $\alpha \in(0,1)$ (see F. Sachweh [21: pp. 16 and $52-54]$ ).

The continuity and Lipschitz continuity of an autonomous Nemytskij operator are completely characterized in the next two theorems. Thereby, Theorem 2.3 shows that there is no sense to assume $F$ satisfies a (global) Lipschitz condition on $H^{\alpha}[a, b]$. Only local Lipschitz continuity makes sense.

Theorem 2.2. The autonomous Nemytskij operator $F$ generated by $f$ maps the space $H^{\alpha}[a, b]$ continuously into itself if and only if $f \in C^{1}(\mathbb{R})$.

Proof. 1. We begin with the sufficiency of the condition $f \in C^{1}(\mathbb{R})$. Due to Theorem 2.1Fu $=f \circ u \in H^{\alpha}[a, b]$ holds for all $u \in H^{\alpha}[a, b]$. To show the continuity of $F: H^{\alpha}[a, b] \rightarrow H^{\alpha}[a, b]$ let there be given a sequence $\left(u_{n}\right) \subset H^{\alpha}[a, b]$ converging to $u_{0} \in H^{\alpha}[a, b]$. Since obviously $f \circ u_{n} \rightarrow f \circ u_{0}$ in $C[a, b]$ it remains to show that

$$
h_{\alpha}\left(f \circ u_{n}-f \circ u_{0}\right) \longrightarrow 0 .
$$

For any $n \in \mathbb{N}$ we define

$$
\Delta_{n}(t, s)=\frac{\left|f\left(u_{0}(t)\right)-f\left(u_{0}(s)\right)-f\left(u_{n}(t)\right)+f\left(u_{n}(s)\right)\right|}{|t-s|^{\alpha}} \quad(t, s \in[a, b], t \neq s) .
$$

Using the Lagrange formula

$$
f(x)-f(y)=(x-y) \int_{0}^{1} f^{\prime}(y+\tau(x-y)) d \tau \quad(x, y \in \mathbb{R})
$$

we may verify that

$$
\begin{aligned}
\Delta_{n}(t, s) \leq & h_{\alpha}\left(u_{0}\right) \int_{0}^{1} \mid f^{\prime}\left(u_{0}(s)+\tau\left(u_{0}(t)-u_{0}(s)\right)\right) \\
& -f^{\prime}\left(u_{n}(s)+\tau\left(u_{n}(t)-u_{n}(s)\right)\right) \mid d \tau \\
& +h_{\alpha}\left(u_{0}-u_{n}\right) \int_{0}^{1}\left|f^{\prime}\left(u_{n}(s)+\tau\left(u_{n}(t)-u_{n}(s)\right)\right)\right| d \tau
\end{aligned}
$$


for all $t, s \in[a, b]$ and all $n \in \mathbb{N}$. Since if $n \rightarrow \infty$ the first integrand tends to zero uniformly on $[a, b] \times[a, b] \times[0,1]$ and since the second integrand is uniformly bounded on the same set, we get (2.3).

2. Now we prove the necessity of $f \in C^{1}(\mathbb{R})$. Again due to Theorem 2.1 we know $f \in \operatorname{Lip}_{\text {loc }}(\mathbb{R})$. Hence, both

$$
L_{1}(x)=\liminf _{\xi \rightarrow 0} \frac{f(x+\xi)-f(x)}{\xi} \quad \text { and } \quad L_{2}(x)=\limsup _{\xi \rightarrow 0} \frac{f(x+\xi)-f(x)}{\xi}
$$

are finite for all $x \in \mathbb{R}$ and, in virtue of the Rademacher Theorem (sec, e.g., [28: p. 50]), $L_{1}(x)=L_{2}(x)=f^{\prime}(x)$ for almost all $x \in \mathbb{R}$. Suppose there exists a point $x_{0} \in \mathbb{R}$ at which $f$ is not differentiable, i.e. $L_{1}\left(x_{0}\right)<L_{2}\left(x_{0}\right)$. We denote by $\left(\xi_{n}\right),\left(\eta_{n}\right) \subset \mathbb{R}$ two zero sequences with

$$
L_{1}\left(x_{0}\right)=\lim _{n \rightarrow \infty} \frac{f\left(x_{0}+\xi_{n}\right)-f\left(x_{0}\right)}{\xi_{n}} \quad \text { and } \quad L_{2}\left(x_{0}\right)=\lim _{n \rightarrow \infty} \frac{f\left(x_{0}+\eta_{n}\right)-f\left(x_{0}\right)}{\eta_{n}}
$$

and by $\left(\delta_{n}\right) \subset \mathbb{R}$ a further zero sequence such that $f$ has in all points $x_{0}+\delta_{n}$ a finite derivative $f^{\prime}\left(x_{0}+\delta_{n}\right)$. Without loss of generality, for all $n \in \mathbb{N}$ we may assume $\xi_{n}>0$ or $\xi_{n}<0$ and $\eta_{n}>0$ or $\eta_{n}<0$. First assuming $\xi_{n}>0$ for all $n$ we consider $u_{n}$ defined by

$$
u_{n}(t)=(t-a)^{\alpha}+x_{0}+\delta_{n} \quad(t \in[a, b], n \in \mathbb{N}) .
$$

Obviously, $u_{n} \in H^{\alpha}[a, b]$, and if $n \rightarrow \infty$, then, in $H^{\alpha}[a, b], u_{n} \rightarrow u_{0} \in H^{\alpha}[a, b]$ where $u_{0}$ is defined by $u_{0}(t)=(t-a)^{\alpha}+x_{0} \quad(t \in[a, b])$. So, by our assumption, $f \circ u_{n} \rightarrow f \circ u_{0}$ in $H^{\alpha}[a, b]$. Taking $\varepsilon=\frac{L_{2}\left(x_{0}\right)-L_{1}\left(x_{0}\right)}{4}$ we find a natural number $n_{0}=n(\varepsilon)$ such that $h_{\alpha}\left(f \circ u_{n}-f \circ u_{0}\right) \leq \varepsilon$ for all $n \geq n_{0}$ which implies

$$
\begin{aligned}
& \mid f\left((t-a)^{\alpha}+x_{0}+\delta_{n}\right)-f\left((s-a)^{\alpha}+x_{0}+\delta_{n}\right) \\
& \quad-f\left((t-a)^{\alpha}+x_{0}\right)+f\left((s-a)^{\alpha}+x_{0}\right)|\leq \varepsilon| \dot{t}-\left.s\right|^{\alpha}
\end{aligned}
$$

for all $t, s \in[a, b]$ and all $n \geq n_{0}$. Substituting'here $t=a+\xi_{m}^{1 / \alpha}$ with $m \in \mathbb{N}$ so large that $t \in[a, b]$, and $s=a$, we get

$$
\left|\frac{f\left(x_{0}+\delta_{n}+\xi_{m}\right)-f\left(x_{0}+\delta_{n}\right)}{\xi_{m}}-\frac{f\left(x_{0}+\xi_{m}\right)-f\left(x_{0}\right)}{\xi_{m}}\right| \leq \varepsilon
$$

for all $n \geq n_{0}$ and $m$ sufficiently large. If in this inequality $m \rightarrow \infty$ we arrive at

$$
\left|f^{\prime}\left(x_{0}+\delta_{n}\right)-L_{1}\left(x_{0}\right)\right| \leq \varepsilon \quad\left(n \geq n_{0}\right) .
$$

If $\xi_{n}<0$ for all $n$, the same arguments but with $u_{n}$ defined by $u_{n}(t)=-(b-t)^{\alpha}+x_{0}+\delta_{n}$ and later $t=b-\left(-\xi_{m}\right)^{1 / \alpha}, s=b$ lead to the same estimate (2.5). Completely in the same way one can show that also $\left|f^{\prime}\left(x_{0}+\delta_{n}\right)-L_{2}\left(x_{0}\right)\right| \leq \varepsilon$ for all $n \geq n_{0}$. So we have

$$
\begin{aligned}
L_{2}\left(x_{0}\right)-L_{1}\left(x_{0}\right) & \leq\left|L_{2}\left(x_{0}\right)-f^{\prime}\left(x_{0}+\delta_{n}\right)\right|+\left|f^{\prime}\left(x_{0}+\delta_{n}\right)-L_{1}\left(x_{0}\right)\right| \\
& \leq 2 \varepsilon \\
& <L_{2}\left(x_{0}\right)-L_{1}\left(x_{0}\right)
\end{aligned}
$$

for all $n \geq n_{0}$ which is a contradiction. Consequently, the generating function $f$ has at each point $x \in \mathbb{R}$ a finite derivative. Because of (2.5) can now be verified for any $x_{0} \in \mathbb{R}$, any zero sequence $\left(\delta_{n}\right) \subset \mathbb{R}$, and any $\dot{\varepsilon}>0$, the continuity of $f^{\prime}$ at $x_{0}$ is also proved, i.e. $f \in C^{1}(\mathbb{R})$ as claimed 
In the proof of the next two theorems we make use of the following lemma. Its proof is quite elementary and hence omitted (see [8]).

Lemma 2.2. Let $h \in C([a, b] \times[0,1])$ and $\alpha \in(0,1]$ be given. If there exists $a$ positive constant $L$ such that

$$
|h(t, \tau)-h(s, \tau)| \leq L|t-s|^{\alpha} \quad(t, s \in[a, b], \tau \in[0,1])
$$

then for the parameter integral $g(t)=\int_{0}^{1} h(t, \tau) d \tau \quad(t \in[a, b])$

$$
g \in H^{\alpha}[a, b] \text { with } h_{\alpha}(g) \leq L \quad \text { and } \quad\|g\|_{H^{\alpha}[a, b]} \leq \int_{0}^{1}\|h(\cdot ; \tau)\|_{H^{\alpha}[a, b]} d \tau
$$

Theorem 2.3. If the autonomous Nemytskij operator $F$ generated by $f$ maps the space $H^{\alpha}[a, b]$ into itself, then:

(a) $F$ is locally Lipschitz continuous if and only if $f \in L_{i p}^{1}(\mathbb{R})$.

(b) $F$ is (globally) Lipschitz continuous if and only if there are two constants $c_{1}, c_{2} \in$ $\mathbb{R}$ such that $f(x)=c_{1} x+c_{2}$ for all $x \in \mathbb{R}$.

Proof. (a)/1. Let $f \in \operatorname{Liploc}_{1}(\mathbb{R})$ and let $F_{1}$ denote the Nemytskij operator generated by the derivative $f^{\prime}$. In virtue of Theorem 2.1 and Corollary $2.1 F_{1}$ is a bounded operator acting in $H^{\alpha}[a, b]$. Because of the Lagrange formula (2.4)

$$
F u-F v=(u-v) \int_{0}^{1} F_{1}(v+\tau(u-v)) d \tau \quad\left(u, v \in H^{\alpha}[a, b]\right)
$$

holds and by means of Lemma 2.2 and Lemma $2.1 /(\mathrm{a})$ we get the estimation

$$
\|F u-F v\|_{H^{\alpha}[a, b]} \leq\|u-v\|_{H^{\alpha}[a, b]} \int_{0}^{1}\left\|F_{1}(v+\tau(u-v))\right\|_{H^{\circ}[a, b]} d \tau
$$

for all $u, v \in H^{\alpha}[a, b]$ which gives the local Lipschitz continuity of $F$.

(a)/2. Assuming now $F \in \operatorname{Liploc}_{\text {loc }}\left(H^{\alpha}[a, b]\right)$, by Theorem 2.2 we have $f \in C^{1}(\mathbb{R})$. So it remains to show the local Lipschitz continuity of $f^{\prime}$. Let $x_{0} \in \mathbb{R}$ be fixed and $u_{0} \in H^{\alpha}[a, b]$ defined by. $u_{0}(t)=(t-a)^{\alpha}+x_{0} \quad(t \in[a, b])$. By our assumption, we find two constants $L_{0}>0$ and $\delta_{0}>0$ such that

$$
\|F u-F v\|_{H^{\circ}[a, b]} \leq L_{0}\|u-v\|_{H^{\alpha}[a, b]} \quad\left(u, v \in B\left(u_{0}, \delta_{0}\right) \subset H^{\alpha}[a, b]\right) .
$$

Clearly, for any $x \in B\left(x_{0}, \delta_{0}\right) \subset \mathbb{R}$ the function $u_{x}$ defined by $u_{x}(t)=(t-a)^{\alpha}+x(t \in$ $[a, b])$ belongs to $B\left(u_{0}, \delta_{0}\right) \subset H^{\alpha}[a, b]$. So we get

$$
\left\|F u_{x}-F u_{y}\right\|_{H^{\circ}[a, b]} \leq L_{0}|x-y| \quad\left(x, y \in B\left(x_{0}, \delta_{0}\right) \subset \mathbb{R}\right)
$$


and, consequently,

$$
\frac{\left|f\left((t-a)^{\alpha}+x\right)-f\left((t-a)^{\alpha}+y\right)-f\left((s-a)^{\alpha}+x\right)+f\left((s-a)^{\alpha}+y\right)\right|}{|t-s|^{\alpha}} \leq L_{0}|x-y|
$$

for all $t, s \in[a, b]$ and all $x, y \in B\left(x_{0}, \delta_{0}\right) \subset \mathbb{R}$. For $s=a$ we obtain eventually

$$
\left|\frac{f\left(x+(t-a)^{\alpha}\right)-f(x)}{(t-a)^{\alpha}}-\frac{f\left(y+(t-a)^{\alpha}\right)-f(y)}{(t-a)^{\alpha}}\right| \leq L_{0}|x-y|
$$

for all $t \in[a, b]$ and all $x, y \in B\left(x_{0}, \delta_{0}\right) \subset \mathbb{R}$. Letting $t \rightarrow a$ we come to

$$
\left|f^{\prime}(x)-f^{\prime}(y)\right| \leq L_{0}|x-y| \quad\left(x, y \in B\left(x_{0}, \delta_{0}\right) \subset \mathbb{R}\right)
$$

which proves the desired property.

(b) Here we have to prove only that the (global) Lipschitz continuity of $F$ in $H^{\alpha}[a, b]$ implies the linearity of the generating function $f$. In $H^{\alpha}[a, b]$ let us take the norm $\|u\|_{H^{a}[a, b]}=|u(a)|+h_{\alpha}(u)$ equivalent to the norm given in (1.1). Let $\tau ; \sigma \in[a, b]$ with $\tau>\sigma$ and $x, y, \xi, \eta \in \mathbb{R}$ be fixed. Consider two functions $u, v \in H^{\alpha}[a, b]$ defined by

$$
u(t)= \begin{cases}x & \text { if } a \leq t \leq \sigma \\ \frac{\xi-x}{\tau-\sigma}(t-\sigma)+x & \text { if } \sigma<t<\tau \\ \xi & \text { if } \tau \leq t \leq b\end{cases}
$$

and

$$
v(t)= \begin{cases}y & \text { if } a \leq t \leq \sigma \\ \frac{\eta-y}{\tau-\sigma}(t-\sigma)+y & \text { if } \sigma<t<\tau \\ \eta & \text { if } \tau \leq t \leq b\end{cases}
$$

Then

$$
\|u-v\|_{H^{\alpha}[a, b]}=|x-y|+\frac{|\xi-\eta-x+y|}{|\tau-\sigma|^{\alpha}} .
$$

Since $F$ is Lipschitz continuous in $H^{\alpha}\{a, b]$, there exists a constant $L>0$ such that

$$
h_{\alpha}(f \circ u-f \circ v) \leq L\|u-v\|_{H^{\alpha}[a, b]} \quad\left(u, v \in H^{\alpha}[a, b]\right) .
$$

Substituting here the functions $u, v$ defined above we get

$$
|f(x)-f(y)-f(\xi)+f(\eta)| \leq L\left(|x-y||\tau-\sigma|^{\alpha}+|\xi-\eta-x+y|\right)
$$

which, for $y=x+h$ and $\eta=\xi+h$ with $h \in \mathbb{R}$ different from zero, results in

$$
\left|\frac{f(x+h)-f(x)}{h}-\frac{f(\xi+h)-f(\xi)}{h}\right| \leq L|\tau-\sigma|^{\alpha} \text {. }
$$

Since by Theorem 2.2 we know that $f \in C^{1}(\mathbb{R})$, we obtain $\left|f^{\prime}(x)-f^{\prime}(\xi)\right| \leq L|\tau-\sigma|^{\alpha}$. Therefore $f^{\prime}(x)=f^{\prime}(\xi)$ for all $x, \xi \in \mathbb{R}$, which implies our assertion 
Finally, we give a necessary and sufficient differentiability criterion.

Theorem 2.4. The autonomous Nemytskij operator $F$ generated by $f$ is continuously Fréchet differentiable in $H^{\alpha}[a, b]$ if and only if $f \in C^{2}(\mathbb{R})$, where its Fréchet derivative $F^{\prime}(u)$ at $u \in H^{\alpha}[a, b]$ is given by

$$
F^{\prime}(u) v=\left(f^{\prime} \circ u\right) v
$$

for all $v \in H^{\alpha}[a, b]$.

Proof. 1. First assume $f \in C^{2}(\mathbb{R})$. Again let $F_{1}$ denote the Nemytskij operator generated by $f^{\prime}$. Due to Theorem 2.2 , we know that $F_{1} \in C\left(H^{\alpha}[a, b]\right)$. Like (2.6) one can verify that

$$
\left\|F(u+v)-F u-F_{1}(u) v\right\|_{H^{\alpha}[a, b]} \leq\|v\|_{H^{a}[a, b]} \int_{0}^{1}\left\|F_{1}(u+\tau v)-F_{1} u\right\|_{H^{\alpha}[a, b]} d \tau
$$

for all $u, v \in H^{\alpha}[a, b]$. Let now $u \in H^{\alpha}[a, b]$ be fixed. Then for any $\varepsilon>0$ there is $\delta>0$ such that

$$
\left\|F_{1}(u+\tau v)-F_{1} u\right\|_{H^{\circ}[a, b]}<\varepsilon
$$

for all $v \in B(0, \delta) \subset H^{\alpha}[a, b]$ and all $\left.\tau \in[0,1]\right)$. Hence, from (2.8)

$$
\left\|F(u+v)-F u-F_{1}(u) v\right\|_{H^{\alpha}[a, b]} \leq \varepsilon\|v\|_{H^{\alpha}[a, b]}
$$

for all $\left.v \in B(0, \delta) \subset H^{\alpha}[a, b]\right)$ follows. Since evidently the map $v \mapsto F_{1}(u) v$ is linear and bounded in $H^{\alpha}[a, b]$, we see that the operator $F$ is Fréchet differentiable at $u \in H^{\alpha}[a, b]$, and its Fréchet derivative at $u$ is given by $F_{1} u$, i.e. relation (2.7) holds. Furthermore, we have

$$
\left\|F^{\prime}(u)-F^{\prime}(v)\right\|_{\mathcal{C}\left(H^{\circ}\{a, b]\right)} \leq\left\|F_{1} u-F_{1} v\right\|_{H^{\alpha}[a, b]} \quad\left(v \in H^{\alpha}[a, b]\right),
$$

showing the continuity of $F^{\prime}: H^{\alpha}[a, b] \rightarrow \mathcal{L}\left(H^{\alpha}[a, b]\right)$ at $u \in H^{\alpha}[a, b]$. So we have proved that $F \in C^{1}\left(H^{\alpha}[a, b]\right)$.

2. Now we show that $F \in C^{1}\left(H^{\alpha}[a, b]\right)$ implies $f \in C^{2}(\mathbb{R})$ and its Fréchet derivative has the form (2.7). First, because of Theorem 2.2, we know that $f \in C^{1}(\mathbb{R})$. To verify (2.7) we fix an arbitrary $u \in H^{\alpha}[a, b]$. Then for any $\varepsilon>0$ there is $\delta>0$ such that

$$
\left\|F(u+\tau v)-F u-\tau F^{\prime}(u) v\right\|_{H^{a}\{a, b]} \leq \varepsilon|\tau|\|v\|_{H^{\alpha}[a, b]}
$$

and, in particular,

$$
\left|\frac{f(u(t)+\tau v(t))-f(u(t))}{\tau}-\left(F^{\prime}(u) v\right)(t)\right| \leq \varepsilon\|v\|_{H^{\circ}[a, b]}
$$

for all $v \in H^{\alpha}[a, b], 0 \neq \tau \in \mathbb{R}$ with $|\tau|\|v\|_{H^{a}[a, b]} \leq \delta$ and all $t \in[a, b]$. If in the last inequality $\tau \rightarrow 0$, we obtain

$$
\left|f^{\prime}(u(t)) v(t)-\left(F^{\prime}(u) v\right)(t)\right| \leq \varepsilon\|v\|_{H^{\alpha}[a, b]}
$$


for all $v \in H^{\alpha}[a, b]$ and all $t \in[a, b]$ which implies the validity of (2.7). Putting there $v=1$ we get $f^{\prime} \circ u \in H^{\alpha}[a, b]$ for all $u \in H^{\alpha}[a, b]$. Furthermore,

$$
\begin{aligned}
\left\|f^{\prime} \circ u-f^{\prime} \circ v\right\|_{H^{\alpha}[a, b]} & =\left\|F^{\prime}(u) 1-F^{\prime}(v) 1\right\|_{H^{\circ}[a, b]} \\
& \leq\left\|F^{\prime}(u)-F^{\prime}(v)\right\|_{\mathcal{L}\left(H^{a}[a, b]\right)} \quad\left(u, v \in H^{\alpha}[a, b]\right) .
\end{aligned}
$$

This means the Nemytskij operator gencrated by the function $f^{\prime}: \mathbb{R} \rightarrow \mathbb{R}$ maps $H^{\alpha}[a, b]$ continuously into itself. Again in virtue of Theorem $2.2 f^{\prime} \in C^{1}(\mathbb{R})$ which completes the proof

We want to mention that particularly for Theorem 2.2 and Theorem 2.4 (but also for Theorem $2.3 /(a))$ there are well-known criteria even for generalized Hölder spaces (see J. Appell and P. P. Zabrejko [2: Chapter 7]). Our proofs are independent of these somehow sophisticated criteria. The sufficient parts of the theorems just mentioned were proved in M. Goebel [8], their necessary parts seem to be new. However, compare P. Drábek [7] concerning Theorem 2.2, and R. Chiappinelli and R. Nugari [6] for the case of non-autonomous Nemytskij operators. Theorem $2.3 /(\mathrm{b})$ was first proved in $\mathrm{J}$. Matkowski [15] for $\alpha=1$, in A. Matkowska [13] for $\alpha \in(0,1)$ and rediscovered in J. Appell, E. De Pascale and P. P. Zabrejko [1]. Further results concerning globally Lipschitzian Nemytskij operators in different function spaces can be found in $[14,16$, 17]. Locally Lipschitz continuous Nemytskij operators in spaces of bounded $\alpha$-Hölder continuous functions defined on $\mathbb{R}$ were investigated by E. P. Sobolevskij [23, 24].

\section{Some auxiliary results}

The study of $F u=f \circ u$ in $H^{k+\alpha}[a, b],(k, \alpha) \in \mathbb{N} \times(0,1]$, first raises the question whether or not $f \circ u$ belongs to $H^{k+\alpha}[a, b]$ if $u$ is an element of the same space. In other words, we need for the $k$-th derivative of the composed function $f \circ u$ a convenient formula, by means of which we may easily answer this and other questions. Such a formula is given below in Lemma 3.3 showing that we have to study also certain nonlinear differential operators. At first, however, we give in Lemma 3.1 some further properties of Hölder and Lipschitz continuous functions required in the subsequent sections.

\section{Lemma 3.1.} $C^{1}[a, b]$

(a) The inclusion $C^{1}[a, b] \subset$ Lip $[a, b]$ holds, where $h_{1}(u)=\left\|u^{\prime}\right\|_{C[a, b]}$ for all $u \in$

(b) For any pairs $(k, \alpha),(l, \beta) \in \mathbb{Z}_{+} \times(0,1]$ with $l+\beta<k+\alpha$ the space $H^{k+\alpha}[a, b]$ is continuously embedded into $H^{i+\beta}[a, b]$.

(c) There exists a constant $c>0$ such that $\left\|D^{i} u\right\|_{H^{\alpha}[a, b]} \leq c\|u\|_{H^{k+\alpha}[a, b]}$ for each $u \in H^{k+\alpha}[a, b]$ and $i \in\{0,1, \ldots, k\}$.

(d) For any $u, v \in H^{k+\alpha}[a, b]$ we have $u v \in H^{k+\alpha}[a, b]$, and there exists a constant $c>0$ such that $\|u v\|_{H^{k+\alpha}[a, b]} \leq c\|u\|_{H^{k+a}[a, b]}\|v\|_{H^{k+a}[a, b]}$ for all $u, v \in H^{k+\alpha}[a, b]$.

Proof. (a) Due to the mean value theorem, for any $u \in C^{1}[a, b]$ and any $s, t \in[a, b]$ with $s \neq t$ there is some $\tau \in[a, b]$ such that $|u(t)-u(s)|=\left|u^{\prime}(\tau)\right||t-s|$. Hence we have 
$u \in \operatorname{Lip}[a, b]$ with $h_{1}(u) \leq\left\|u^{\prime}\right\|_{C[a, b]}$. To show the converse inequality let $t_{0} \in[a, b]$ be such that $\left|u^{\prime}\left(t_{0}\right)\right|=\left\|u^{\prime}\right\|_{C[a, b]}$. Then

$$
h_{1}(u) \geq \frac{\left|u\left(t_{0}\right)-u(t)\right|}{\left|t_{0}-t\right|} \quad\left(t \in[a, b], t \neq t_{0}\right)
$$

and if $t \rightarrow t_{0}$, we get $h_{1}(u) \geq\left|u^{\prime}\left(t_{0}\right)\right|$ which proves the wanted equality.

(b) If $l=k$, then $\beta<\alpha$ and the statement follows via Lemma $2.1 /(\mathrm{b})$. If $l+1 \leq k$, the inclusion $H^{k+\alpha}[a, b] \subset H^{l+\beta}[a, b]$ is obvious, and because of Lemma 2.1/(b) and property (a) for any $u \in H^{k+\alpha}[a, b]$ we have

$$
\|u\|_{H^{i+o}[a, b]}=\sum_{i=0}^{l}\left\|D^{i} u\right\|_{C[a, b]}+h_{\beta}\left(D^{l} u\right) \leq c \sum_{i=0}^{l+1}\left\|D^{i} u\right\|_{C[a ; b]} \leq c\|u\|_{H^{k+\alpha}[a, b]}
$$

showing the continuity of this embedding.

(c) Let $u \in H^{k+\alpha}[a, b]$ be fixed. Because of (1.2) the statement is evident if $i=k$. In the case of $i<k$ we use Lemma $2.1 /(\mathrm{b})$ and property (a) proved above to get

$$
\begin{aligned}
\left\|D^{i} u\right\|_{H^{\circ}[a, b]} & \leq\left\|D^{i} u\right\|_{C[a, b]}+(b-a)^{1-\alpha} h_{1}\left(D^{i} u\right) \\
& =\left\|D^{i} u\right\|_{C[a, b]}+(b-a)^{1-\alpha}\left\|D^{i+1} u\right\|_{C[a, b]}
\end{aligned}
$$

from which the statement follows.

(d) If $u, v \in H^{k+\alpha}[a, b]$, then $D^{k-j} u, D^{j} v \in H^{\alpha}[a, b](j=0, \ldots, k)$ and due to the Leibniz formula

$$
D^{k}(u v)=\sum_{j=0}^{k}\left(\begin{array}{l}
k \\
j
\end{array}\right) D_{. .}^{k-j} u D^{j} v
$$

and Lemma $2.1 /(a)$ we get $D^{k}(u v) \in H^{\alpha}[a, b]$, which means $u v \in H^{k+\alpha}[a, b]$. Furthermore, we have

$$
\|u v\|_{H^{k+a}[a, b]} \leq\|u v\|_{H^{\circ}[a, b]}+\sum_{i=1}^{k}\left\|D^{i}(u v)\right\|_{H^{\alpha}[a, b]}
$$

Taking advantage of (3.1), Lemma $2.1 /(a)$ and statement (c) just proved we obtain

$$
\begin{aligned}
\|u v\|_{H^{k+a}[a, b]} & \left.\leq\|u v\|_{H^{a}[a, b]}+\sum_{i=1}^{k} \sum_{j=0}^{i}\left(\begin{array}{l}
i \\
j
\end{array}\right) \| D^{i-j} u D^{j} v\right) \|_{H^{\circ}[a, b]} \\
& \leq c\left(1+\sum_{i=1}^{k} \sum_{j=0}^{i}\left(\begin{array}{l}
i \\
j
\end{array}\right)\right)\|u\|_{H^{k+a}[a, b]}\|v\|_{H^{k+a}[a, b]}
\end{aligned}
$$

where the constant $c$ is the same as in statement (c) 
Let $\mathcal{P}_{k}(k \in \mathbb{N})$ be the set of all differential operators $P$ defined by

$$
P u=p\left(u^{\prime}, \ldots, u^{(k)}\right) \quad\left(u \in C^{k}[a, b]\right)
$$

where $p \in C\left(\mathbb{R}^{k}\right)$ is a polynomial of the form

$$
p\left(x_{1}, \ldots, x_{k}\right)=\sum_{i=1}^{m} c_{i}\left(\prod_{j=1}^{k} x_{j}^{a_{i j}}\right) \quad\left(\left(x_{1}, \ldots, x_{k}\right) \in \mathbb{R}^{k}\right)
$$

with $m, c_{i} \in \mathbb{N}, a_{i j} \in \mathbb{Z}_{+}$and $a_{i_{0} k}>0$ for at least one index $i_{0}$.

We consider $P \in \mathcal{P}_{k}$ as a map defined on $H^{k+\alpha}[a, b]$. To prepare the most important Lemmas 3.3 - 3.5 we prove firstly the following one.

Lemma 3.2. For any $P \in \mathcal{P}_{k}$ there exists $Q \in \mathcal{P}_{k+1}$ such that $D P u=Q u$ for all $u \in C^{k+1}[a, b]$.

Proof. Firstly, for any $k \in \mathbb{N}$ let $a_{1}, \ldots, a_{k-1} \in \mathbb{Z}_{+}$and $a_{k} \in \mathbb{N}$ be given. Using induction it can be shown that there exists $Q \in \mathcal{P}_{k+1}$ such that

$$
D\left(\prod_{j=1}^{k}\left(D^{j} u\right)^{a_{j}}\right)=Q u \quad\left(u \in C^{k+1}[a, b]\right) .
$$

Then for an arbitrary $P \in \mathcal{P}_{k}$ generated by $p \in C\left(\mathbb{R}^{k}\right)$ as given in (3.2) we have

$$
D P u=\sum_{i=1}^{m} c_{i} D\left(\prod_{j=1}^{k}\left(D^{j} u\right)^{a_{i j}}\right) \quad\left(u \in C^{k+1}[a, b]\right)
$$

where $a_{i_{0} k}>0$ for at least one index $i_{0}$. Since there exists $Q_{i_{0}} \in \mathcal{P}_{k+1}$ with

$$
D\left(\prod_{j=1}^{k}\left(D^{j} u\right)^{a_{i_{0} j}}\right)=Q_{i_{0}} u \quad\left(u \in C^{k+1}[a, b]\right)
$$

the desired statement follows

Lemma 3.3. For each $f \in C^{k}(\mathbb{R})$ there are $k$ operators $P_{i} \in \mathcal{P}_{k-i+1}(i=1, \ldots, k)$ such that

$$
D^{k}(f \circ u)=\sum_{i=1}^{k}\left(f^{(i)} \circ u\right) P_{i} u
$$

for all $u \in C^{k}[a, b]$.

Proof. The assertion will be proved by induction. If $f \in C^{1}(\mathbb{R})$ and $u \in C^{1}[a, b]$, formula (3.4) is true because of $D(f \circ u)=\left(f^{\prime} \circ u\right) P_{1} u$ with $P_{1} \in \mathcal{P}_{1}$ generated by $p_{1}\left(x_{1}\right)=x_{1}$. Assume for $k \in \mathbb{N}$ the statement to be true with certain $\widetilde{P}_{i} \in \mathcal{P}_{k-i+1} \quad(i=$ 
$1, \ldots, k)$. Then in virtue of Lemma 3.2 there are $\widetilde{Q}_{i} \in \mathcal{P}_{k-i+2}$ with $D \widetilde{P}_{i} u=\widetilde{Q}_{i} u$ for all $u \in C^{k-i+2}[a, b] \quad(i=1, \ldots, k)$. If now $f \in C^{k+1}(\mathbb{R})$ and $u \in C^{k+1}[a, b]$, then

$$
\begin{aligned}
D^{k+1}(f \circ u) & =D\left(D^{k}(f \circ u)\right) \\
& =\sum_{i=1}^{k} D\left[\left(f^{(i)} \circ u\right) \widetilde{P}_{i} u\right] \\
& =\sum_{i=1}^{k}\left(f^{(i+1)} \circ u\right) u^{\prime} \widetilde{P}_{i} u+\sum_{i=1}^{k}\left(f^{(i)} \circ u\right) \widetilde{Q}_{i} u \\
& =\sum_{i=1}^{k+1}\left(f^{(i)} \circ u\right) P_{i} u
\end{aligned}
$$

where in the last equation we have put

$$
\left.\begin{array}{rl}
P_{1} u & =\widetilde{Q}_{1} u \\
P_{i} u & =u^{\prime} \widetilde{P}_{i-1} u+\widetilde{Q}_{i} u \quad(i=2, \ldots, k) \\
P_{k+1} u & =u^{\prime} \widetilde{P}_{k} u .
\end{array}\right\}
$$

Since $P_{i} \in \mathcal{P}_{k-i+2} \quad(i=1, \ldots, k+1)$, the statement is proved for $k+1$

Lemma 3.4. Each differential operator $P \in \mathcal{P}_{k}$ maps $H^{k+\alpha}[a, b]$ into $H^{\alpha}[a, b]$ and is locally Lipschitz continuous.

Proof. By the definition of $P \in \mathcal{P}_{k}$ it is evident that $P u \in H^{\alpha}[a, b]$ for each $u \in H^{k+\alpha}[a, b]$. So if $u_{0} \in H^{k+\alpha}[a, b]$ and $\delta>0$ are fixed, the statement follows after showing that there is another constant $c=c\left(u_{0}, \delta\right)>0$ such that

$$
\|P u-P v\|_{H^{a}[a, b]} \leq c\|u-v\|_{H^{k+\alpha}[a, b]}
$$

for all $u, v \in B\left(u_{0}, \delta\right) \subset H^{k+\alpha}[a, b]$. Let $P$ be generated by $p \in C\left(\mathbb{R}^{k}\right)$ as given in (3.2). Then using the identity

$$
\prod_{j=1}^{k} x_{j}-\prod_{j=1}^{k} y_{j}=\sum_{j=1}^{k}\left[\left(\prod_{l=1}^{j-1} x_{l}\right)\left(x_{j}-y_{j}\right)\left(\prod_{l=j+1}^{k} y_{l}\right)\right] \quad\left(x_{j}, y_{j} \in \mathbb{R}, k \in \mathbb{N}\right)
$$

and Lemma $2.1 /(\mathrm{a})$, for arbitrary $u, v \in H^{k+\alpha}[a, b]$ we get

$$
\begin{aligned}
\|P u-P v\|_{H^{\circ}[a, b]}= & \left\|\sum_{i=1}^{m} c_{i}\left[\prod_{j=1}^{k}\left(D^{j} u\right)^{a_{i j}}-\prod_{j=1}^{k}\left(D^{j} v\right)^{a_{i j}}\right]\right\|_{H^{a}[a, b]} \\
\leq & c \sum_{i=1}^{m} \sum_{j=1}^{k}\left[\left(\prod_{l=1}^{j-1}\left\|D^{l} u\right\|_{H^{\circ}[a, b]}^{a_{i l}}\right) \|\left(D^{j} u\right)^{a_{i j}}\right. \\
& \left.-\left(D^{j} v\right)^{a_{i j}} \|_{H^{a}[a, b]}\left(\prod_{l=j+1}^{k}\left\|D^{l} v\right\|_{H^{\circ}[a, b]}^{a_{i l}}\right)\right] .
\end{aligned}
$$


Because of Lemma $3.1 /(\mathrm{c})$ we have for all $u \in B\left(u_{0}, \delta\right) \subset H^{k+\alpha}[a, b]$

$$
\left\|D^{l} u\right\|_{H^{a}[a, b]} \leq c \quad \text { and } \quad\left\|\left(D^{j} u\right)^{a_{i j}}-\left(D^{j} v\right)^{a_{i j}}\right\|_{H^{a}[a, b]} \leq c\|u-v\|_{H^{k+a}[a, b]}
$$

where in proving the second estimate we may take advantage of the elementary identity

$$
x^{k}-y^{k}=(x-y) \sum_{i=0}^{k-1} x^{k-i-1} y^{i} \quad(x, y \in \mathbb{R}, k \in \mathbb{N})
$$

Combining the last two estimates with (3.6) we obtain the desired property (3.5)

Lemma 3.5. If $i \in\{1, \ldots, k-1\}$ is fixed, then each differential operator $P \in \mathcal{P}_{k-i}$ maps $H^{k+\alpha}[a, b]$ into $H^{1+\alpha}[a, b]$ and is locally Lipschitz continuous.

Proof. Let $i \in\{1, \ldots, k-1\}$ and $P \in \mathcal{P}_{k-i}$ be fixed. By the definition of the class $\mathcal{P}_{k-i}, P u \in H^{1+\alpha}[a, b]$ for each $u \in H^{k+\alpha}[a, b]$. In virtue of Lemma 3.2 there exists another differential operator $Q \in \mathcal{P}_{k-i+1}$ with $D P u=Q u$ for all $u \in C^{k-i+1}[a, b]$. Thus we have

$$
\begin{aligned}
\|P u-P v\|_{H^{1+a}[a, b]} & \leq\|P u-P v\|_{H^{a}[a, b]}+\|D(P u-P v)\|_{H^{a}[a, b]} \\
& =\|P u-P v\|_{H^{a}[a, b]}+\|Q u-Q v\|_{H^{a}[a, b]}
\end{aligned}
$$

for all $u, v \in H^{k+\alpha}[a, b]$. Applying Lemma 3.4 to $P$ and $Q$ for any $u_{0} \in H^{k+\alpha}[a, b]$ and any $\delta>0$ we find some constants $c>0$ and $\delta>0$ such that, for all $u, v \in B\left(u_{0}, \delta\right) \subset$ $H^{k+\alpha}[a, b]$,

$$
\begin{aligned}
& \|P u-P v\|_{H^{\alpha}[a, b]} \leq c\|u-v\|_{H^{k-i+a}[a, b]} \\
& \|Q u-Q v\|_{H^{\alpha}[a, b]} \leq c\|u-v\|_{H^{k-i+s+a[a, b]}} .
\end{aligned}
$$

Observe that $M=B\left(u_{0}, \delta\right) \subset H^{k+\alpha}[a, b]$ implies $M \subset B\left(u_{0}, \delta\right) \subset H^{k-j+\alpha}[a, b]$ for any $j=1, \ldots, k-1$. From the given estimates the desired local Lipschitz continuity of $P: H^{k+\alpha}[a, b] \rightarrow H^{1+\alpha}[a, b]$ immediately follows

Lemma 3.3 shows that $D^{k}(f \circ u)$ can be written as a sum of products, where in the products the term $f^{(i)} \circ u$ is one factor and in the other $u^{(k-i+1)}$ is the highest derivative of $u$ which occurs. Taking into account these auxiliary results we are now in a position to prove new properties of the Nemytskij operator in $\operatorname{Lip}^{k}[a, b]$ and $H^{k+\alpha}[a, b]$, respectively.

\section{The Nemytskij operator in $\operatorname{Lip}^{k}[a, b]$}

In this section we consider the autonomous Nemytskij operator $F$ in $\operatorname{Lip}^{k}[a, b]$ with $k \in \mathbb{N}$. The theorems below are analogous to those given in Section 2 for the space $H^{\alpha}[a, b]$ with $\alpha \in(0,1]$. Since $H^{1}[a, b]=\operatorname{Lip}[a, b]=\operatorname{Lip} p^{0}[a, b]$, the statements are also true for $k=0$. 
Theorem 4.1. The autonomous Nemytskij operator $F$ generated by $f$ maps the space Lip ${ }^{k}[a, b]$ into itself if and only if $f \in \operatorname{Lip}_{l o c}^{k}(\mathbb{R})$.

Proof. 1. We show the sufficiency of $f \in \operatorname{Lip}_{l o c}^{k}(\mathbb{R})$. Let $u \in L_{i p^{k}}[a, b]$ be given. Obviously, $f \circ u \in C^{k}[a, b]$ and relation (3.4) holds with certain $P_{i} \in \mathcal{P}_{k-i+1}$. Since for $i=1, \ldots, k$ we have

$$
f^{(i)} \in \operatorname{Liploc}_{l}(\mathbb{R}) \quad \text { and } \quad \operatorname{Lip}^{k}[a, b] \subset \operatorname{Lip}^{k-i+1}[a, b] \subset \operatorname{Lip}[a, b]
$$

from Theorem 2.1 we get $f^{(i)} \circ u \in \operatorname{Lip}[a, b]$ and from Lemma 3.4 also $P_{i} u \in \operatorname{Lip}[a, b]$. Hence by formula (3.4) $D^{k}(f \circ u) \in \operatorname{Lip}[a, b]$ follows which means $f \circ u \in \operatorname{Lip}^{k}[a, b]$.

2. Let now $f: \mathbb{R} \rightarrow \mathbb{R}$ be such that $f \circ u \in \operatorname{Lip}^{k}[a, b]$ for all $u \in \operatorname{Lip}^{k}[a, b]$. For arbitrary $x_{0} \in \mathbb{R}$ and $\delta=x_{0}-a-\frac{b-a}{2}$ the linear function $u_{0}$ defined by $u_{0}(t)=$ $t+\delta(t \in[a, b])$ is an element of $\operatorname{Lip}^{k}[a, b]$ and therefore also $\left(f \circ u_{0}\right)(t)=f(t+\delta)$. Hence $f(\cdot+\delta) \in C^{k}[a, b]$, that means $f \in C^{k}\left[x_{0}-\frac{b-a}{2}, x_{0}+\frac{b-a}{2}\right]$. Moreover, $D^{k}\left(f \circ u_{0}\right)=$ $f^{(k)} \circ u_{0} \in \operatorname{Lip}[a, b]$, i.e. there exists a constant $L_{0}>0$ such that

$$
\left|f^{(k)}\left(u_{0}(t)\right)-f^{(k)}\left(u_{0}(s)\right)\right| \leq L_{0}|t-s|=L_{0}\left|u_{0}(t)-u_{0}(s)\right| \quad(t, s \in[a, b])
$$

which implies $\left|f^{(k)}(x)-f^{(k)}(y)\right| \leq L_{0}|x-y|$ for all $x, y \in B\left(x_{0}, \frac{b-a}{2}\right) \subset \mathbb{R}$. So we have $f \in L i p^{k}\left[x_{0}-\frac{b-a}{2}, x_{0}+\frac{b-a}{2}\right]$ and consequently $f \in L_{i p}^{k}(\mathbb{R})$

Corollary 4.1. Each autonomous Nemytskij operator $F$ acting in Lip $[a, b]$ is bounded.

Theorem 4.2. The autonomous Nemytskij operator $F$ generated by $f$ maps the space $L i p^{k}[a, b]$ continuously into itself if and only if $f \in C^{k+1}(\mathbb{R})$.

Proof. 1. Firstly we assume $f \in C^{k+1}(\mathbb{R})$. Because of $C^{k+1}(\mathbb{R}) \subset L i p_{\text {loc }}^{k}(\mathbb{R})$ and Theorem 4.1, the Nemytskij operator $F$ generated by $f$ maps $L i p^{k}[a, b]$ into itself. To show its continuity let the sequence $\left(u_{n}\right) \subset \operatorname{Lip}^{k}[a, b]$ converge to $u \in \operatorname{Lip}^{k}[a, b]$. By Lemma $3.1 /(b)$ this implies

$$
u_{n} \longrightarrow u \quad \text { in } \operatorname{Lip}^{i}[a, b] \text { for all } i=0, \ldots, k-1 \text {. }
$$

Applying Lemma 3.3 we find

$$
\begin{aligned}
\left\|F u_{n}-F u\right\|_{\left.L i p^{k} \mid a, b\right]} \leq & \left\|f \circ u_{n}-f \circ u\right\|_{L_{i p[a, b]}} \\
& +\sum_{i=1}^{k}\left\|D^{i}\left(f \circ u_{n}\right)-D^{i}(f \circ u)\right\|_{L i p[a, b]} \\
\leq & \left\|f \circ u_{n}-f \circ u\right\|_{L i p[a, b]} \\
& +\sum_{i=1}^{k} \sum_{j=1}^{i}\left\|\left(f^{(j)} \circ u_{n}\right) P_{i j} u_{n}-\left(f^{(j)} \circ u\right) P_{i} u\right\|_{L i p[a, b]}
\end{aligned}
$$

with certain $P_{i j} \in \mathcal{P}_{i-j+1}$. Since by assumption $f^{(j)} \in C^{1}(\mathbb{R})(j=0, \ldots, k)$, Theorem 2.2 implies $f^{(j)} \circ u_{n} \rightarrow f^{(j)} \circ u$ in $\operatorname{Lip}[a, b]$, and since in virtue of (4.1) and Lemma 3.4 
$P_{i j} u_{n} \rightarrow P_{i j} u$ in $\operatorname{Lip}[a, b]$, we obtain $F u_{n} \rightarrow F u$ in $\operatorname{Lip}^{k}[a, b]$ which proves the continuity of $F$ at the point $u \in \operatorname{Lip}^{k}[a, b]$.

2. Let now $F \in C\left(\operatorname{Lip}^{k}[a, b]\right)$. By. Theorem 4.1 we have $f \in \operatorname{Lip}_{\text {loc }}^{k}(\mathbb{R})$ which implies $D^{k} f \in L_{i p l o c}(\mathbb{R})$, and it remains to show that $D^{k} f \in C^{1}(\mathbb{R})$. Like in the second part of the proof to Theorem 2.2 , for each $x_{0} \in \mathbb{R}$ there exists a sequence $\left(x_{n}\right) \subset \mathbb{R}$ converging to $x_{0}$ and $f^{(k)}$ differentiable at each $x_{n}$. Consider the functions $u_{n} \in \operatorname{Lip}^{k}[a, b]$ for each $n \in \mathbb{Z}_{+}$defined by $u_{n}(t)=t-\xi+x_{n} \quad(t \in[a, b], \xi \in(a, b))$. Evidently, $D^{k}\left(f \circ u_{n}\right)=$ $f^{(k)} \circ u_{n}$ and $u_{n} \rightarrow u_{0}$ in $\operatorname{Lip}^{k}[a, b]$ if $n \rightarrow \infty$. Since $F$ is continuous at $u_{0}$, for arbitrary $\varepsilon>0$ there is an $n_{0}=n_{0}(\varepsilon) \in \mathbb{N}$ with $\left\|F u_{n}-F u_{0}\right\|_{L i p^{k}[a, b]} \leq \varepsilon$ for all $n \geq n_{0}$, and therefore

$$
\frac{\left|f^{(k)}\left(u_{n}(t)\right)-f^{(k)}\left(u_{0}(t)\right)-f^{(k)}\left(u_{n}(s)\right)+f^{(k)}\left(u_{0}(s)\right)\right|}{|t-s|} \leq \varepsilon
$$

for all $t, s \in[a, b]$ and all $n \geq n_{0}$. In particular, for $s=\xi$ we obtain

$$
\left|\frac{f^{(k)}\left(t-\xi+x_{n}\right)-f^{(k)}\left(x_{n}\right)}{t-\xi}-\frac{f^{(k)}\left(t-\xi+x_{0}\right)-f^{(k)}\left(x_{0}\right)}{t-\xi}\right| \leq \varepsilon
$$

for all $t \in[a, b]$ and all $n \geq n_{0}$. Supposing $f^{(k)}$ to be not differentiable at $x_{0}$ we can find two zero sequences $\left(\tau_{n}\right),\left(\theta_{n}\right) \subset \mathbb{R}$ and two reell numbers $L_{1}, L_{2}$ such that

$$
L_{1}=\lim _{m \rightarrow \infty} \frac{f^{(k)}\left(x_{0}+\tau_{m}\right)-f^{(k)}\left(x_{0}\right)}{\tau_{m}}<\lim _{m \rightarrow \infty} \frac{f^{(k)}\left(x_{0}+\theta_{m}\right)-f^{(k)}\left(x_{0}\right)}{\theta_{m}}=L_{2} .
$$

If in estimate (4.3) we put $t=\xi+\tau_{m}, m$ sufficiently large, and if afterwards $m \rightarrow \infty$, we find

$$
\left|f^{(k+1)}\left(x_{n}\right)-L_{1}\right| \leq \varepsilon \quad\left(n \geq n_{0}\right)
$$

and in the same way $\left|f^{(k+1)}\left(x_{n}\right)-L_{2}\right| \leq \varepsilon$ for all $n \geq n_{0}$. Thus we come to $\left|L_{1}-L_{2}\right| \leq \varepsilon$, which contradicts $L_{1}<L_{2}$. Hence $f^{(k)}$ is differentiable at any point $x_{0} \in \mathbb{R}$. The continuity of $f^{(k+1)}$ follows from (4.4) (see the proof of Theorem 2.2)

Theorem 4.3. The autonomous Nemytskij operator $F$ generated by $f$ is a locally Lipschitz continuous map from $\operatorname{Lip}^{k}[a, b]$ into itself if and only if $f \in \operatorname{Lip}_{\text {loc }}^{k+1}(\mathbb{R})$.

Proof. 1. Firstly let us assume $f \in \operatorname{Lip}_{l o c}^{k+1}(\mathbb{R})$. By formula (4.2), with $u_{n}$ replaced by $v$, for all $u, v \in \operatorname{Lip}^{k}[a, b]$

$$
\begin{aligned}
\|F u-F v\|_{L_{i p^{k}[a, b]} \leq} & \|f \circ u-f \circ v\|_{L i p[a, b]} \\
& +\sum_{i=1}^{k} \sum_{j=1}^{i}\left[\left\|P_{i j} v\right\|_{L i p[a, b]}\left\|f^{(j)} \circ u-f^{(j)} \circ v\right\|_{L i p[a, b]}\right. \\
& \left.+\left\|f^{(j)} \circ u\right\|_{L i p[a, b]}\left\|P_{i j} u-P_{i j} v\right\|_{L i p[a, b]}\right]
\end{aligned}
$$

holds where $P_{i j} \in \mathcal{P}_{i-j+1}$. The desired local Lipschitz continuity of $F: L_{i p^{k}}[a, b] \rightarrow$ $\operatorname{Lip}^{k}[a, b]$ follows now from Theorem 2.3/(a) applied to the Nemytskij operators $F, F_{1}$, $\ldots, F_{k}$ generated by $f, f^{\prime}, \ldots, f^{(k)}$, respectively, Lemma 3.4 and finally Lemma $3.1 /(\mathrm{b})$. 
2. If, on the other hand, we assume $F: \operatorname{Lip}^{k}[a, b] \rightarrow \operatorname{Lip}{ }^{k}[a, b]$ to be locally Lipschitz continuous, then due to Theorem 4.2 the generating function $f$ belongs to $C^{k+1}(\mathbb{R})$. So we have to show only that $f^{(k+1)}$ is locally Lipschitz continuous on $\mathbb{R}$. To prove this for any $x \in \mathbb{R}$ we define $u_{x}$ by $u_{x}(t)=t-a+x \quad(t \in[a, b])$. In particular, for fixed $x_{0} \in \mathbb{R}$ we set $u_{0}=u_{x_{0}}$. Then $\left\|u_{x}-u_{y}\right\|_{L_{i p^{k}}(a, b]}=|x-y|$ for all $x, y \in \mathbb{R}$ and hence, for any $\delta>0, u_{x} \in B\left(u_{0}, \delta\right) \subset \operatorname{Lip}^{k}[a, b]$ provided $\left|x-x_{0}\right|<\delta$. So by our assumption there are two constants $L_{0}>0$ and $\delta_{0}>0$ such that $\left\|F u_{x}-F u_{y}\right\|_{L i p^{k}[a, b]} \leq L_{0}|x-y|$ for all $x, y \in B\left(x_{0}, \delta_{0}\right) \subset \mathbb{R}$ which because of $D^{k}\left(f \circ u_{x}\right)=f^{(k)} \circ u_{x}$ implies

$$
\left|\frac{f^{(k)}(t-a+x)-f^{(k)}(t-a+y)-f^{(k)}(s-a+x)+f^{(k)}(s-a+y)}{t-s}\right| \leq L_{0}|x-y|
$$

for all $t, s \in[a, b]$ and all $x, y \in B\left(x_{0}, \delta_{0}\right) \subset \mathbb{R}$. For $s=a$ this estimate gives

$$
\left|\frac{f^{(k)}(x+t-a)-f^{(k)}(x)}{t-a}-\frac{f^{(k)}(y+t-a)-f^{(k)}(y)}{t-a}\right| \leq L_{0}|x-y|
$$

for all $t \in[a, b]$ and all $x, y \in B\left(x_{0}, \delta_{0}\right) \subset \mathbb{R}$ from which, if $t \rightarrow a$, we get $\mid f^{(k+1)}(x)-$ $f^{(k+1)}(y)\left|\leq L_{0}\right| x-y \mid$ for all $x, y \in B\left(x_{0}, \delta_{0}\right) \subset \mathbb{R}$. This shows the claimed local Lipschitz continuity of $f^{(k+1)}$ on $\mathbb{R}$

Theorem 4.4. The autonomous Nemytskij operator $F$ generated by $f$ is continuously Fréchet differentiable in $\operatorname{Lip}^{k}[a, b]$ if and only if $f \in C^{k+2}(\mathbb{R})$. Its Fréchet derivative $F^{\prime}(u)$ at $u \in \operatorname{Lip}^{k}[a, b]$ is given by

$$
F^{\prime}(u) v=\left(f^{\prime} \circ u\right) v
$$

for all $v \in \operatorname{Lip}^{k}[a, b]$

Proof. That $F \in C^{1}\left(\operatorname{Lip}^{k}[a, b]\right)$ implies $f \in C^{k+2}(\mathbb{R})$ and the validity of $(4.5)$ can be shown in the same way as in the proof of Theorem 2.4 for $k=0$. So assuming conversely $f \in C^{k+2}(\mathbb{R})$ we are going to show $F \in C^{1}\left(\operatorname{Lip}^{k}[a, b]\right)$ and formula (4.5). Due to Theorem 4.2 the Nemytskij operator $F_{1}$ defined by $F_{1} u=f^{\prime} \circ u\left(u \in \operatorname{Lip} p^{k}[a, b]\right)$ belongs to $C\left(\operatorname{Lip}^{k}[a, b]\right)$. Hence for any $u, v \in \operatorname{Lip}^{k}[a, b]$ the function $\varphi(\tau)=F_{1}(u+\tau v)$ maps continuously the interval $[0,1]$ into $\operatorname{Lip}^{k}[a, b]$ and thus is also integrable. Let $u \in \operatorname{Lip}^{k}[a, b]$ and $\varepsilon>0$ be fixed. Then there exists some $\delta>0$ such that

$$
\left\|\int_{0}^{1}\left(F_{1}(u+\tau v)-F_{1} u\right) d \tau\right\|_{L i p^{k}[a, b]} \leq \int_{0}^{1}\left\|F_{1}(u+\tau v)-F_{1} u\right\|_{L i p^{k}[a, b]} d \tau<\varepsilon
$$

for all $v \in B(0, \delta) \subset \operatorname{Lip}^{k}[a, b]$. The classical Lagrange formula (2.4) yields

$$
f(u(t)+v(t))-f(u(t))-f^{\prime}(u(t)) v(t)=v(t) \int_{0}^{1}\left(f^{\prime}(u(t)+\tau v(t))^{-}-f^{\prime}(u(t))\right) d \tau
$$


for all $t \in[a, b]$ and all $u, v \in \operatorname{Lip}^{k}[a, b]$. Since here the parameter integral is the same as the integral on the left-hand side of estimate (4.6), we can apply Lemma $3.1 /(d)$. So taking advantage of (4.6) we get

$$
\begin{aligned}
\left\|F(u+v)-F u-F_{1}(u) v\right\|_{L i p^{k}[a, b]} & \leq c\|v\|_{L i p^{k}[a, b]}\left\|\int_{0}^{1}\left(F_{1}(u+\tau v)-F_{1,} u\right) d \tau\right\|_{L i p^{k}[a, b]} . \\
& \leq c \varepsilon\|v\|_{L i p^{k}[a, b]}
\end{aligned}
$$

for all $v \in B(0, \delta) \subset \operatorname{Lip}^{k}[a, b]$ which means the Fréchet derivative of $F$ at $u$ is given by $F_{1} u=f^{\prime} \circ u \in \mathcal{L}\left(\operatorname{Lip}^{k}[a, b]\right)$. Since its continuity is obvious the theorem is proved

These results are quite satisfactory and in a certain sense final. By no means the same can be said concerning those of the next section.

\section{The Nemytskij operator in $H^{k+\alpha}[a, b]$}

Now we come to the most interesting case, namely to $H^{k+\alpha}[a, b]$ with $(k, \alpha) \in \mathbb{N} \times(0,1)$. Not very much papers are dealing with the Nemytskij operator in this general Hölder space (see J. Appell and P. P. Zabrejko [2: Section 8.3]). However, let us mention the introductory Section 1.3 of the monograph E. Wegert [26], which is devoted to nonautonomous Nemytskij operators acting in $H^{k+\alpha}(\Omega)\left(\Omega \subset \cdot \mathbb{R}^{n}\right)$, but also in $C^{k}(\Omega)$ and Sobolev spaces. Sufficient acting conditions combined with boundedness, continuity and differentiability conditions are given. Naturally, our sufficient conditions given below are sharper than those which one gets by specifying Wegert's results.

Again we begin with an acting condition, which in this section will be the only necessary and sufficient condition. Note that Theorem 5.1 is not true for $k=0$, however for $\alpha=1$ (compare with Theorem 2.1 and Theorem 4.1).

Theorem 5.1. The autonomous Nemytskij operator $F$ generated by $f$ maps the space $H^{k+\alpha}[a, b],(k, \alpha) \in \mathbb{N} \times(0,1)$, into itself if and only if $f \in H_{\text {loc }}^{k+\alpha}(\mathbb{R})$.

Proof. 1. Let first $f \in H_{\text {loc }}^{k+\alpha}(\mathbb{R})$. Since for any $i=1, \ldots, k$ and any $u \in H^{k+\alpha}[a, b]$ the derivative $D^{i}(f \circ u)$ belongs to $C[a, b]$, it remains to show $D^{k}(f \circ u) \in H^{\alpha}[a, b]$. By Lemma 3.3

$$
D^{k}(f \circ u)=\sum_{i=1}^{k}\left(f^{(i)} \circ u\right) P_{i} u \quad\left(u \in H^{k+\alpha}[a, b]\right)
$$

holds with certain differential operators $P_{i} \in \mathcal{P}_{k-i+1}$. Because of Lemma $3.1 /(\mathrm{b})$ and Lemma 3.4 we have $P_{i} u \in H^{\alpha}[a, b]$ for $i=1, \ldots, k$, and because of Theorem 2.1 we have $f^{(i)} \circ u \in H^{\alpha}[a, b]$ for $i=1, \ldots, k-1$. Consider now $f^{(k)} \circ u$ for any fixed $u \in H^{k+\alpha}[a, b]$. Choosing $r>0$ so large that $|u(t)|<r$ for each $t \in[a, b]$, we find a constant $L_{r}>0$ such that

$$
\left|f^{(k)}(x)-f^{(k)}(y)\right| \leq L_{r}|x-y|^{\alpha} \quad(x, y \in[-r, r]),
$$

hence, by the mean value theorem,

$$
\left|f^{(k)}(u(t))-f^{(k)}(u(s))\right| \leq L_{r}|u(t)-u(s)|^{\alpha} \leq L_{r}\left\|u^{\prime}\right\|_{C[a, b]}^{\alpha}|t-s|^{\alpha}
$$


for all $t, s \in[a, b]$. Thus we also have $f^{(k)} \circ u \in H^{\alpha}[a, b]$ and formula (5.1) yields $D^{k}(f \circ u) \in H^{\alpha}[a, b]$ for each $u \in H^{k+\alpha}[a, b]$, which shows that $F$ generated by $f \in$ $H_{l o c}^{k+\alpha}(\mathbb{R})$ maps $H^{k+\alpha}[a, b]$ into itself.

2. The proof of the necessity of $f \in H_{l o c}^{k+\alpha}(\mathbb{R})$ is almost the same as in Theorem 4.1 (for $\alpha=1$ ) and therefore omitted

Corollary 5.1. Each autonomous Nemytskij operator $F$ acting in $H^{k+\alpha}[a, b],(k, \alpha)$ $\in \mathbb{N} \times(0,1)$, is bounded.

We change over to continuity conditions for $F$ in $H^{k+\alpha}[a, b]$. Like before, continuity of $F$ is not already implied by its acting in $H^{k+\alpha}[a, b]$. As a simple illustrating example in $H^{1+\alpha}[0,1]$, with $\alpha \in(0,1)$, one may take the Nemytskij.operator $F$ generated by the function $f$ defined by

$$
f(x)= \begin{cases}\frac{x^{1+\alpha}}{1+\alpha} & \text { if } x>0 \\ 0 & \text { if } x \leq 0\end{cases}
$$

Looking to what we have found concerning continuity in the foregoing sections, we could expect that the autonomous Nemytskij operator $F$ generated by $f$ is continuous in $H^{k+\alpha}[a, b]$ if and only if $f \in C^{k+1}(\mathbb{R})$. But in the following Theorem 5.2, which is an improvement of the corresponding theorem announced in [11], we have proved a sufficient condition weaker than the inclusion $f \in V^{k+1}(\mathbb{R})$. Note, on the other hand, that this sufficient continuity criterion seems to be only a very little touch more restrictive than the necessary condition from Theorem 5.1 .

Theorem 5.2. The autonomous Nemytskij operator $F$ generated by $f$ maps the space $H^{k+\alpha}[a, b],(k, \alpha) \in \mathbb{N} \times(0,1)$, continuously into itself if, for any $r>0$, there exists $\beta \in(\alpha, 1]$ such that $f \in H^{k+\beta}[-r, r]$.

Proof. Since the assumption implies $f \in H_{\text {loc }}^{k+\alpha}(\mathbb{R})$, in virtue of Theorem 5.1 the related Nemytskij operator $F$ maps $H^{k+\alpha}[a, b]$ into itself. At first we prove the continuity of this map in the case of $k=1$. So let $\left(u_{n}\right)$ be an arbitrary sequence in $H^{1+\alpha}[a, b]$ converging to $u_{0} \in H^{1+\alpha}[a, b]$. We choose $r \geq 1$ and $n_{0} \in \mathbb{N}$ such that

$$
\left\|u_{n}\right\|_{H^{1+\alpha}[a, b]} \leq r \quad \text { and } \quad\left\|u_{n}-u_{0}\right\|_{H^{1+\alpha}[a, b]} \leq 1
$$

for each $n \geq n_{0}$. By assumption $f \in \operatorname{Lip}[-r, r]$ and $f^{\prime} \in H^{\beta}[-r, r]$. Let $L$ and $L_{\beta}$ denote the Lipschitz and Hölder constant, respectively. Then we have the estimate

$$
\begin{aligned}
\| F u_{n} & -F u_{0} \|_{H^{1+a}[a, b]} \\
= & \left\|f \circ u_{n}-f \circ u_{0}\right\|_{C[a, b]}+\left\|\left(f^{\prime} \circ u_{n}\right) u_{n}^{\prime}-\left(f^{\prime} \circ u_{0}\right) u_{0}^{\prime}\right\|_{H^{\circ}[a, b]} \\
\leq & L\left\|u_{n}-u_{0}\right\|_{C[a, b]}+\left\|f^{\prime} \circ u_{0}\right\|_{H^{\circ}[a, b]}\left\|u_{n}^{\prime}-u_{0}^{\prime}\right\|_{H^{a}[a, b]} \\
& +r\left\|f^{\prime} \circ u_{n}-f^{\prime} \circ u_{0}\right\|_{H^{\circ}[a, b]} \\
\leq & c\left\|u_{n}-u_{0}\right\|_{H^{1+\circ}[a, b]}+r L_{\beta}\left\|u_{n}-u_{0}\right\|_{C[a, b]}^{\beta}+r h_{\alpha}\left(f^{\prime} \circ u_{n}-f^{\prime} \circ u_{0}\right) \\
\leq & c\left\|u_{n}-u_{0}\right\|_{H^{1+a}[a, b]}^{\beta}+r h_{\alpha}\left(f^{\prime} \circ u_{n}-f^{\prime} \circ u_{0}\right)
\end{aligned}
$$

for all $n \geq n_{0}$. To show that the second term tends to zero provided $n \rightarrow \infty$, for each $n \geq n_{0}$ we introduce $\Delta_{n}(t, s)$ defined by

$$
\Delta_{n}(t, s)=\left|f^{\prime}\left(u_{n}(t)\right)-f^{\prime}\left(u_{0}(t)\right)-f^{\prime}\left(u_{n}(s)\right)+f^{\prime}\left(u_{0}(s)\right)\right| \quad(t, s \in[a, b]) .
$$


Because of $f^{\prime} \in H^{\beta}[-r, r]$ we have

$$
\begin{aligned}
\Delta_{n}(t, s) & \leq L_{\beta}\left(\left|u_{n}(t)-u_{0}(t)\right|^{\beta}+\left|u_{n}(s)-u_{0}(s)\right|^{\beta}\right) \\
& \leq 2 L_{\beta}\left\|u_{n}-u_{0}\right\|_{H^{1+\alpha}[a, b]}^{\beta} \\
& \leq 2 r^{\beta} L_{\beta}\left\|u_{n}-u_{0}\right\|_{H^{1+\alpha}[a, b]}^{\beta}
\end{aligned} \quad(t, s \in[a, b]) .
$$

On the other hand, again using that $f^{\prime} \in H^{\beta}[-r, r]$ and additionally Lemma $3.1 /(a)$, we find

$$
\begin{aligned}
\Delta_{n}(t, s) & \leq L_{\beta}\left(\left|u_{n}(t)-u_{n}(s)\right|^{\beta}+\left|u_{0}(t)-u_{0}(s)\right|^{\beta}\right) \\
\cdot & \leq L_{\beta}\left(\left\|u_{n}^{\prime}\right\|_{C[a, b]}^{\beta}+\left\|u_{0}^{\prime}\right\|_{C[a, b]}^{\beta}\right)|t-s|^{\beta} \quad(t, s \in[a, b]) . \\
& \leq 2 r^{\beta} L_{\beta}|t-s|^{\beta}
\end{aligned}
$$

We put $\gamma=\alpha \beta^{-1}$ which implies $\gamma \in(0,1), \beta \gamma=\alpha$, and $\beta(1-\gamma)=\beta-\alpha>0$. By (5.3) and (5.4) we obtain

$$
\begin{aligned}
\Delta_{n}(t, s) & =\left(\Delta_{n}(t, s)\right)^{1-\gamma}\left(\Delta_{n}(t, s)\right)^{\gamma} \\
& \leq 2 r^{\beta} L_{\beta}\left\|u_{n}-u_{0}\right\|_{H^{1+\alpha}[a, b]}^{\beta-\alpha}|t-s|^{\alpha} \quad(t, s \in[a, b]) .
\end{aligned}
$$

Hence for all $n \geq n_{0}$ we have the estimate

$$
h_{\alpha}\left(f^{\prime} \circ u_{n}-f^{\prime} \circ u_{0}\right) \leq 2 r^{\beta} L_{\beta}\left\|u_{n}-u_{0}\right\|_{H^{1+\alpha}\{a, b\}}^{\beta-\alpha},
$$

showing together with (5.2) that $F u_{n} \rightarrow F u_{0}$ in $H^{1+\alpha}[a, b]$ as claimed.

Consider now the general case $k \geq 2$. Again let $\left(u_{n}\right)$ be a sequence in $H^{k+\alpha}[a, b]$ converging to $u_{0} \in H^{k+\alpha}[a, b]$. Observe that this implies $u_{n} \rightarrow u_{0}$ in $\operatorname{Lip}^{k-2}[a, b]$ and $u_{n} \rightarrow u_{0}$ in $H^{1+\alpha}\{a, b]$. Since by assumption $f \in C^{k-1}(\mathbb{R})$, Theorem 4.2 yields

$$
f \circ u_{n} \longrightarrow f \circ u_{0} \quad \text { in } \operatorname{Lip}^{k-2}[a, b] \text {. }
$$

Furthermore, by assumption, for any $i=1, \ldots, k-1$ and $r>0$ we can find $\beta \in(\alpha, 1]$ with $f^{(i)} \in H^{1+\beta}[-r, r]$. Thus, by what we have just proved for $k=1$,

$$
f^{(i)} \circ u_{n} \longrightarrow f^{(i)} \circ u_{0} \quad \text { in } H^{1+\alpha}[a, b] \text { for } i=1, \ldots, k-1 \text {. }
$$

Finally, because of Lemma 3.5, for any differential operator $P_{i} \in \mathcal{P}_{k-i}(i=1, \ldots, k-1)$ we have

$$
P_{\mathrm{i}} u_{\mathrm{n}} \longrightarrow P_{\mathrm{i}} u_{0} \quad \text { in } H^{1+\alpha}[a, b] .
$$

We estimate for any $n \in \mathbb{N}$ now the norm of $F u_{n}-F u_{0}$ in $H^{k+\alpha}[a, b]$ :

$$
\begin{aligned}
\| F u_{n} & -F u_{0} \|_{H^{k+\alpha}[a, b]} \\
\leq & \left\|f \circ u_{n}-f \circ u_{0}\right\|_{L i p^{k-2}[a, b]}+\left\|D^{k-1}\left(f \circ u_{n}-f \circ u_{0}\right)\right\|_{H^{1+a}[a, b]} \\
\leq & \left\|f \circ u_{n}-f \circ u_{0}\right\|_{L i p^{k-2}[a, b]} \\
& \quad+\sum_{i=1}^{k-1}\left\|\left(f^{(i)} \circ u_{n}\right) P_{i} u_{n}-\left(f^{(i)} \circ u_{0}\right) P_{i} u_{0}\right\|_{H^{1+a}[a, b]}
\end{aligned}
$$

where $P_{i} \in \mathcal{P}_{k-i}$ (see Lemma 3.3). Because of (5.5) - (5.8) the proof is complete 
The situation is similar in the case of local Lipschitz continuity and continuous Fréchet differentiability of $F$. Taking into account the results from the previous sections one could expect $F$ to be locally Lipschitz continuous or continuously Fréchet differentiable in $H^{k+\alpha}[a, b]$ if and only if the generating function $f$ belongs to $\operatorname{Lip}_{\text {loc }}^{k+1}(\mathbb{R})$ or $C^{k+2}(\mathbb{R})$, respectively. As it can be seen in the subsequent two theorems these conditions are indeed sufficient. However, we could not verify their necessity. So, there are still differences between the sufficient and the necessary conditions.

Theorem 5.3.

(a) The autonomous Nemytskij operator $F$ generated by $f$ is a locally Lipschitz continuous map in $H^{k+\alpha}[a, b],(k, \alpha) \in \mathbb{N} \times(0,1)$, if $f \in \operatorname{Lip}_{\text {loc }}^{k+1}(\mathbb{R})$.

(b) If conversely $F$ is a locally Lipschitz continuous map in $H^{k+\alpha}[a, b],(k, \alpha) \in$ $\mathbb{N} \times(0,1)$, then $f \in H_{\text {loc }}^{(k+1)+\alpha}(\mathbb{R})$.

Proof. (a) First let $k=1$. So we suppose $f \in \operatorname{Lip}_{\text {loc }}^{2}(\mathbb{R})$. We fix an arbitrary element $u_{0} \in H^{1+\alpha}[a, b]$ and a number $\delta>0$. Let $r>0$ be so large that $\|u\|_{H^{1+\alpha}[a, b]} \leq r$ for all $u \in B\left(u_{0}, \delta\right) \subset H^{1+\alpha}[a, b]$. Let $L_{r}$ denote the Lipschitz constant of $f$ with respect to the interval $[-r, r]$. Because of $f^{\prime} \in \operatorname{Lip}_{\text {loc }}^{l}(\mathbb{R})$, Theorem 2.3 gives a constant $L>0$ such that

$$
\left.\left\|f^{\prime} \circ u-f^{\prime} \circ v\right\|_{H^{\circ}[a, b]} \leq L\|u-v\|_{H^{\circ}[a, b]} \quad\left(u, v \in B\left(u_{0}, \delta\right) \subset H^{1+\alpha} \mid a, b\right]\right) .
$$

Then we have

$$
\begin{aligned}
\| F u- & F v \|_{H^{1+a}[a, b]} \\
= & \|f \circ u-f \circ v\|_{C[a, b]}+\left\|\left(f^{\prime} \circ u\right) u^{\prime}-\left(f^{\prime} \circ v\right) v^{\prime}\right\|_{H^{a}[a, b]} \\
\leq & L_{r}\|u-v\|_{C[a, b]}+\left\|\left(f^{\prime} \circ u\right)\right\|_{H^{\circ}[a, b]}\left\|u^{\prime}-v^{\prime}\right\|_{H^{\alpha}[a, b]} \\
& +\left\|v^{\prime}\right\|_{H^{\circ}[a, b]}\left\|f^{\prime} \circ u-f^{\prime} \circ v\right\|_{H^{\circ}[a, b]} \\
\leq & c\|u-v\|_{H^{1+a}[a, b]}
\end{aligned}
$$

for all $u, v \in B\left(u_{0}, \delta\right) \subset H^{1+\alpha}[a, b]$ and the statement is proved.

Let now $k \geq 2$. Again we take an arbitrary $u_{0} \in H^{k+\alpha}[a, b]$ and an arbitrary number $\delta>0$. Since the assumption $f \in \operatorname{Lip}_{\text {loc }}^{k+1}(\mathbb{R})$ implies $f \in \operatorname{Lip}_{\text {loc }}^{k-1}(\mathbb{R})$ and $f^{\prime}, \ldots, f^{(k-1)} \in \operatorname{Lip}_{\text {loc }}^{2}(\mathbb{R})$, by Theorem 4.3 and the assertion already proved for $k=1$ we find a constant $c>0$ such that

$$
\|f \circ u-f \circ v\|_{L i p^{k-2}[a, b]} \leq c\|u-v\|_{L i p^{k-2}[a, b]}
$$

and

$$
\left\|f^{(i)} \circ u-f^{(i)} \circ v\right\|_{H^{1+\alpha}[a, b]} \leq c\|u-v\|_{H^{1+\alpha}[a, b]} \quad(i=1, \ldots, k-1)
$$

for each $u, v \in B\left(u_{0}, \delta\right) \subset H^{k+\alpha}[a, b]$. By Lemma 3.5, without loss of generality with the same constant $c$, for any $P_{i} \in \mathcal{P}_{k-i}(i=1, \ldots, k-1)$ we have

$$
\left\|P_{i} u-P_{i} v\right\|_{H^{1+o}[a, b]} \leq c\|u-v\|_{H^{k+a}[a, b]} \quad\left(u, v \in B\left(u_{0}, \delta\right) \subset H^{k+\alpha}[a, b]\right) .
$$


Therefore from estimate (5.8) with $u$ instead of $u_{n}$ and $v$ instead of $u_{0}$ the assertion follows also in this case.

(b) If we suppose now $F \in L_{i p}\left(H^{k+\alpha}[a, b]\right)$, then by Theorem 5.1 we have $f \in$ $C^{k}(\mathbb{R})$. So it is to show that $f^{(k)} \in H_{\text {loc }}^{1+\alpha}(\mathbb{R})$. By assumption, for any. $u_{0} \in H^{k+\alpha}[a, b]$ there exist two constants $L_{0}>0$ and $\delta_{0}>0$ such that

$$
\|F u-F v\|_{H^{k+a}[a, b]} \leq L_{0}\|u-v\|_{H^{k+\alpha}[a, b]}
$$

for all $u, v \in B\left(u_{0}, \delta_{0}\right) \subset H^{k+\alpha}[a, b]$. Firstly as $u_{0}$ we take $u_{0}(t)=t-a+x_{0} \quad(t \in[a, b])$ where $x_{0} \in \mathbb{R}^{\prime}$ is arbitrarily fixed. Since the function $u_{x}$ defined by $u_{x}(t)=t-a+x(t \in$ $[a, b]$ ) belongs to $B\left(u_{0}, \delta_{0}\right) \subset H^{k+\alpha}[a, b]$ if $x \in B\left(x_{0}, \delta_{0}\right) \subset \mathbb{R}$, from (5.9) we obtain

$$
\left|f^{(k)}(t-a+x)-f^{(k)}(t-a+y)\right| \leq\left\|D^{k}\left(f \circ u_{x}-f \circ u_{y}\right)\right\|_{C[a, b]} \leq L_{0}|x-y|
$$

for all $t \in[a, b]$ and all $x, y \in B\left(x_{0}, \delta_{0}\right) \subset \mathbb{R}$ which implies

$$
\left|f^{(k)}(x)-f^{(k)}(y)\right| \leq L_{0}|x-y| \quad\left(x, y \in B\left(x_{0}, \delta_{0}\right) \subset \mathbb{R}\right),
$$

i.e. $f^{(k)} \in \operatorname{Lip}_{l o c}(\mathbb{R})$. Suppose $f^{(k)}$ is not differentiable at $x_{0}$. Then there are two zero sequences $\left(\tau_{n}\right),\left(\theta_{n}\right) \subset \mathbb{R}$ and two constants $L_{1}>0$ and $L_{2}>0$ with $L_{1}<L_{2}$ such that

$$
\begin{aligned}
& L_{1}=\liminf _{h \rightarrow 0} \frac{f^{(k)}\left(x_{0}+h\right)-f^{(k)}\left(x_{0}\right)}{h}=\lim _{n \rightarrow \infty} \frac{f^{(k)}\left(x_{0}+\tau_{n}\right)-f^{(k)}\left(x_{0}\right)}{\tau_{n}}, \\
& L_{2}=\limsup _{h \rightarrow 0} \frac{f^{(k)}\left(x_{0}+h\right)-f^{(k)}\left(x_{0}\right)}{h}=\lim _{n \rightarrow \infty} \frac{f^{(k)}\left(x_{0}+\theta_{n}\right)-f^{(k)}\left(x_{0}\right)}{\theta_{n}} .
\end{aligned}
$$

In (5.9) we take now $u_{0}$ defined by $u_{0}(t)=t-\xi+x_{0} \quad(t \in[a, b])$ with fixed $\xi \in(a, b)$. Furthermore, we define $u_{n}$ by $u_{n}(t)=t-\xi+x_{0}+\tau_{n} .(t \in[\dot{a}, b])$. Let $n_{0}=n_{0}\left(\delta_{0}\right) \in \mathbb{N}$ so large that $\left|\tau_{n}\right|<\delta_{0}$ for all $n \geq n_{0}$ and hence $u_{n} \in B\left(u_{0}, \delta_{0}\right) \subset H^{k+\alpha}[a, b]$. Estimate (5.9) yields

$$
\left\|F u_{n}-F u_{0}\right\|_{H^{k+a}[a, b]} \leq L_{0}\left|\tau_{n}\right| \quad\left(n \geq n_{0}\right) .
$$

In particular, we have

$$
h_{\alpha}\left(D^{k}\left(f \circ u_{n}-f \circ u_{0}\right)\right) \leq L_{0}\left|\tau_{n}\right| \quad\left(\dot{n} \geq n_{0}\right)
$$

and hence

$$
\frac{\left|f^{(k)}\left(t-\xi+x_{0}\right)-f^{(k)}\left(t-\xi+x_{0}+\tau_{n}\right)-f^{(k)}\left(x_{0}\right)+f^{(k)}\left(x_{0}+\tau_{n}\right)\right|}{|t-\xi|^{\alpha}} \leq L_{0}\left|\tau_{n}\right|
$$

valid for all $t \in[a, b]$. Because of $f^{(k)} \in L_{i p l o c}(\mathbb{R})$ and the Rademacher Theorem (see, e.g., [28: p. 50]) there exists a further zero sequence $\left(h_{m}\right) \subset \mathbb{R}$ such that $f^{(k)}$ is differentiable at all points $x_{0}+h_{m}$. Without loss of generality we may assume $\xi+h_{m} \epsilon$ $[a, b]$ for all $m \geq n_{0}$. Hence substituting $t=\xi+h_{m}$ into $(5,10)$ for all $n, m \geq n_{0}$ we get

$$
\left|\frac{f^{(k)}\left(x_{0}+h_{m}+\tau_{n}\right)-f^{(k)}\left(x_{0}+h_{m}\right)}{\tau_{n}}-\frac{f^{(k)}\left(x_{0}+\tau_{n}\right)-f^{(k)}\left(x_{0}\right)}{\tau_{n}}\right| \leq L_{0}\left|h_{m}\right|^{\alpha},
$$


from which

$$
\left|f^{(k+1)}\left(x_{0}+h_{m}\right)-L_{1}\right| \leq L_{0}\left|h_{m}\right|^{\alpha} \quad\left(m \geq n_{0}\right)
$$

follows. Consequently, for any $\varepsilon>0$ there exists an $m_{0}=m_{0}(\varepsilon) \geq n_{0}$ with

$$
\left|f^{(k+1)}\left(x_{0}+h_{m}\right)-L_{1}\right| \leq \varepsilon \quad\left(m \geq m_{0}\right) .
$$

Since the same estimate is true with $L_{2}$ instead of $L_{1}, L_{1}=L_{2}$ follows and hence $f^{(k)}$ is differentiable at $x_{0}$.

It remains to show that $f^{(k+1)} \in H_{\text {loc }}^{\alpha}(\mathbb{R})$. Again let $x_{0} \in \mathbb{R}$ be arbitrary but fixed, $u_{0} \in H^{k+\alpha}[a, b]$ be given by $u_{0}(t)=t-a+x_{0} \quad(t \in[a, b])$ and let $L_{0}>0$ and $\delta_{0}>0$ be two constants such that (5.9) holds. Let $\delta_{1}=\min \left\{\frac{\delta_{0}}{2}, b-a\right\}$. Then $u$ definded by $u(t)=t-a+x+\Delta \cdot(t \in[a, b])$ is an element of the ball $B\left(u_{0}, \delta_{0}\right) \subset H^{k+\alpha}[a, b]$ provided $x \in B\left(x_{0}, \delta_{1}\right) \subset \mathbb{R}$ and $\Delta \in B\left(0, \delta_{1}\right) \subset \mathbb{R}$. Since also $v$ defined by $v(t)=t-a+x \quad(t \in$ $[a, b])$ belongs to $B\left(u_{0}, \delta_{0}\right) \subset H^{k+\alpha}[a, b]$ from (5.9) we obtain

$$
\frac{\left|f^{(k)}(t-a+x+\Delta)-f^{(k)}(t-a+x)-f^{(k)}(x+\Delta)+f^{(k)}(x)\right|}{|t-a|^{\alpha}} \leq L_{0}|\Delta|
$$

valid for all $t \in[a, b]$. Let now $y \in B\left(x_{0}, \delta_{1}\right) \subset \mathbb{R}$ be another arbitrary element. Without loss of generality we may assume $x<y$ and hence $a<a+y-x<b$. Substituting $t=a+y-x$ into the last estimate we find

$$
\left|\frac{f^{(k)}(y+\Delta)-f^{(k)}(y)}{\Delta}-\frac{f^{(k)}(x+\Delta)-f^{(k)}(x)}{\Delta}\right| \leq L_{0}|y-x|^{\alpha}
$$

for all $\Delta \in B\left(0, \delta_{1}\right) \subset \mathbb{R}$. If here $\Delta \rightarrow 0$, we.obtain

$$
\left|f^{(k+1)}(y)-f^{(k+1)}(x)\right| \leq L_{0}|y-x| \quad\left(x, y \in B\left(x_{0}, \delta_{1}\right) \subset \mathbb{R}\right)
$$

showing that $f^{(k+1)}$ belongs to $H_{\text {loc }}^{\alpha}(\mathbb{R})$. This completes the proof

\section{Theorem 5.4.}

(a) The autonomous Nemytskij operator $F$ generated by $f$ is a continuously Fréchet differentiable map in the space $H^{k+\alpha}[a, b],(k, \alpha) \in \mathbb{N} \times(0,1)$, if $f \in C^{k+2}(\mathbb{R})$.

(b) If conversely $F$ is a continuously Fréchet differentiable map in $H^{k+\alpha}[a, b]$, $(k, \alpha) \in \mathbb{N} \times(0,1)$, then $f \in H_{\text {loc }}^{(k+1)+\alpha}(\mathbb{R})$.

. In both cases the Fréchet derivative at any point $u \in H^{k+\alpha}[a, b]$ is given by

$$
F^{\prime}(u) v=\left(f^{\prime} \circ u\right) v
$$

for all $v \in H^{k+\alpha}[a, b]$.

The proof of this theorem is nearly the same as the proofs of the analogous statements in the previous sections and hence omitted. 


\section{References}

[1] Appell, J., De Pascale, E. and P. P. Zabrejko: An application of B. N. Sadouskij's fixed point principle to nonlinear singular equations. Z. Anal. Anw. 6 (1987), $193-208$.

[2] Appell, J. and P. P. Zabrejko: Nonlinear Superposition Operators. Cambridge: University Press 1990

[3] Babajev, A. A.: On the structure of a certain nonlinear operator and its application (in Russian). Azerbajdzh. Gos. Univ. Uchen. Zapiski (1961)4, 13 - 16.

[4] Berkolajko, M. Z.: On a nonlinear operator acting in generalized Hölder spaces (in Russian). Voronezh. Gos. Univ., Trudy Sem. Funk. Anal. (1969)12, 96 - 104.

[5] Björk, J.-E.: On extensions of Lipschitz functions. Arkiv Math. 7 (1968), No. 37, 513 515.

[6] Chiappinelli, R. and R. Nugari: The Nemitskii operator in Hölder spaces: Some necessary and sufficient conditions. J. Lond. Math. Soc. (II. Ser.) 51 (1995), $365-372$.

[7] Drábek, P.: Continuity of Nemytskij's operator in Hölder spaces. Cómm. Math. Univ. Carolinae 16 (1975), 37 - 57.

[8] Goebel, M.: On Fréchet-differentiability of Nemytskij operators acting in Hölder spaces. Glasgow Math. J. 33 (1991), 1 - 5.

[9] Goebel, M.: Continuity and Fréchet-differentiability of Nemytskij operators in Hölder spaces. Monatsh. Math. 113 (1992), 107 - 119.

[10] Goebel, M. and D. Oestreich: Optimal control of a nonlinear singular integral equation arising in electrochemical machining. Z. Anal. Anw. 10 (1991), $73-82$.

[11] M. Goebel and F. Sachweh: The autonomous Nemytskij operator in Hölder spaces. Nonlin. Anal., Theory, Methods \& Applications 30 (1997), No. 1, 513-519.

[12] Lanza de Cristoforis, M.: Higher order differentiability properties of the composition and of the inversion operator. Indag. Math. (N.S.) 5 (1994), $457-482$.

[13] Matkowska, A.: On characterization of Lipschitzian operators of substitution in the class of Hölder's functions. Zeszyty Naukowe Politechniki Lodzkiej 17 (1984), 81 - 85.

[14] Matkowska, A., Matkowski, J. and N. Merentes: Remark on globally Lipschitzian composion operators. Demonstr. Math. 28 (1995), $171-175$.

[15] Matkowski, J.: Functional equations and Nemytskii operators. Funkcialaj Ekvacioj 25 (1982), $127-132$.

[16] Matkowski, J.: On Nemytskii operator. Math. Japonica 33 (1988), 81 - 86.

[17] Matkowski, J.: Lipschitzian composition operators in some function spaces. Nonlin. Anal.: Theory, Methods \& Appl. 30 (1997), $719-726$.

[18] McShane, E. J.: Extension of range of functions. Bull. Am. Math. Soc. 40 (1934), 837 -842 .

[19] Nugari, R.: Continuity and differentiability properties of the Nemytskij operator in Hölder spaces. Glasgow Math. J. 30 (1988), $59-65$.

[20] Nugari, R.: Further remarks on the Nemitskii operator in Hölder spaces. Comm. Math. Univ. Carolinae 34 (1993), 89 - 95.

[21] Sachweh, F.: Der Nemytskijoperator in Hölderräumen. Diplomarbeit. Halle: MartinLuther-Universität Halle-Wittenberg 1995.

[22] Sobolevskij, E. P.: The superposition operator in Hölder spaces (in Russian). Unpublished paper. Dep. VINITI No. 3765-84, Moscow 1984 
[23] Sobolevskij, E. P.: The superpositon operator in Hölder spaces (in Russian). Unpublished paper. Dep. VINITI No. 8802-V85, Moscow 1985.

[24] Valent, T.: Boundary Value Problems of Finite Elasticity. New York - Berlin - Heidel berg: Springer-Verlag 1988.

[25] Walter, W.: Analysis II. Berlin - Heidelberg: Springer-Verlag 1990.

[26] Wegert, E.: Nonlinear Boundary Value Problems for Holomorphic Functions and Singular Integral Equations (Mathematical Research: Vol. 65). Berlin: Akademie-Verlag 1992.

[27] von Wolfersdorf, L.: On the theory of nonlinear singular integral equations of Cauchy type. Math. Meth. Appl. Sci. 7 (1985), 493 - 517.

[28] Ziemer, W. P.: Weakly Differentiable Functions. Sobolev Spaces and Functions of Bounded Variation. New York - Berlin - Heidelberg: Springer-Verlag 1989. 